\title{
Latent Olefin Metathesis Catalysts Featuring
}

\section{Chelating Alkylidenes}

Andrew Hejl, Michael W. Day, and Robert H. Grubbs*

Supporting Information 


\section{Methods and Materials}

Unless otherwise noted all reagents were purchased from Aldrich. 1 and $\mathbf{2}$ were donated by Materia, Inc. $3,{ }^{1} \mathbf{1 2},{ }^{2} \mathbf{1 3},{ }^{3} \mathrm{S1}^{4}$ were prepared according to literature procedures. Anhydrous $\mathrm{CH}_{2} \mathrm{Cl}_{2}$ and $\mathrm{C}_{6} \mathrm{D}_{6}$ were obtained by elution through a solvent column drying system. ${ }^{5} \mathrm{CD}_{2} \mathrm{Cl}_{2}$ was purchased from Cambridge Isotope Laboratories and distilled from $\mathrm{CaH}_{2}$ into a Schlenk tube and degassed. $\mathbf{1 0}$ (Aldrich) was distilled under vacuum prior to use.

\section{Catalyst Synthesis}

General procedure for the synthesis of imines $\mathrm{CH}_{2}=\mathrm{CHCH}_{2} \mathrm{CMe}_{2} \mathrm{CH}_{2} \mathrm{~N}=\mathrm{CHR}(\mathbf{8 a}-\mathbf{b})$

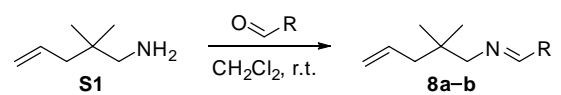

The condensation of 2,2-dimethyl-4-pentenylamine (S1) with various aldehydes was carried out in $\mathrm{CH}_{2} \mathrm{Cl}_{2}$ over activated $4 \AA$ molecular sieves at r.t. for $12 \mathrm{~h}$. The sieves were removed by filtration and the solution concentrated under vacuum to give the desired imines.

$\mathrm{R}=\mathrm{CH}_{2} \mathrm{Ph}(\mathbf{8 a})$

Amine S1 (1.00 mL, $0.78 \mathrm{~g}, 6.9 \mathrm{mmol})$ and benzaldehyde $(0.70 \mathrm{~mL}, 0.73 \mathrm{~g}, 6.9 \mathrm{mmol})$ in $\mathrm{CH}_{2} \mathrm{Cl}_{2}(15 \mathrm{~mL})$ gave $8 \mathbf{a}(0.975 \mathrm{~g}, 4.84 \mathrm{mmol})$ as a clear liquid containing approximately $8 \%$ excess benzaldehyde. Yield: $70 \%$. ${ }^{1} \mathrm{H}$ NMR $\left(\mathrm{CDCl}_{3}, 299.87 \mathrm{MHz}, \delta\right): 8.24(\mathrm{~s}, 1 \mathrm{H}, \mathrm{CH}=\mathrm{N})$, $7.76(\mathrm{~m}, 2 \mathrm{H}, \mathrm{Ph}), 7.42(\mathrm{~m}, 3 \mathrm{H}, \mathrm{Ph}), 5.98-5.82\left(\mathrm{~m}, 1 \mathrm{H}, \mathrm{CH}_{2}=\mathrm{CHCH}_{2}\right), 5.09-5.00(\mathrm{~m}, 2 \mathrm{H}$, $\mathrm{CH}_{2}=\mathrm{CH}$ ), $3.40\left(\mathrm{~s}, 2 \mathrm{H}, \mathrm{CMe}_{2} \mathrm{CH}_{2} \mathrm{~N}\right.$ ), 2.10 (d, $J=7.5 \mathrm{~Hz}, 2 \mathrm{H}$, =CHCH $\left.\mathrm{CMe}_{2}\right), 0.98(\mathrm{~s}, 6 \mathrm{H}$, $\left.\mathrm{CMe}_{2}\right) .{ }^{13} \mathrm{C}\left\{{ }^{1} \mathrm{H}\right\}$ NMR $\left(\mathrm{CDCl}_{3}, 75.41 \mathrm{MHz}, \delta\right): 161.08,136.69,135.66,130.57,128.72,128.26$, 117.18, 72.27, 45.19, 35.48, 25.80. IR $\left(\mathrm{CH}_{2} \mathrm{Cl}_{2}\right.$ soln, $\left.v_{\mathrm{C}=\mathrm{N}}, \mathrm{cm}^{-1}\right): 1647.5$.

$\mathrm{R}=\mathrm{CMe}_{3}(\mathbf{8 b})$

Amine S1 (0.88 mL, $0.69 \mathrm{~g}, 6.1 \mathrm{mmol})$ and trimethylacetaldehyde $(0.72 \mathrm{~mL}, 0.57 \mathrm{~g}, 6.6 \mathrm{mmol})$ in $\mathrm{CH}_{2} \mathrm{Cl}_{2}(15 \mathrm{~mL})$ gave $\mathbf{8 b}(0.634 \mathrm{~g}, 3.50 \mathrm{mmol})$ as a clear liquid. Yield: $58 \%$. ${ }^{1} \mathrm{H}$ NMR $\left(\mathrm{CDCl}_{3}, 300.09 \mathrm{MHz}, \delta\right): 7.44(\mathrm{t}, J=1.5 \mathrm{~Hz}, 1 \mathrm{H}, \mathrm{CH}=\mathrm{N}), 5.91-5.76\left(\mathrm{~m}, 1 \mathrm{H}, \mathrm{CH}_{2}=\mathrm{CHCH}_{2}\right)$, 5.04-4.94 (m, $\left.2 \mathrm{H}, \mathrm{CH}_{2}=\mathrm{CH}\right), 3.12$ (d, $J=0.9 \mathrm{~Hz}, 2 \mathrm{H}, \mathrm{CMe}_{2} \mathrm{CH}_{2} \mathrm{~N}$ ), $1.98(\mathrm{dt}, J=9.0,1.2 \mathrm{~Hz}, 2$ $\left.\left.\mathrm{H},=\mathrm{CHCH}_{2} \mathrm{CMe}_{2}\right), 1.06(\mathrm{~s}, 9 \mathrm{H}, \mathrm{N}=\mathrm{CHCMe})_{3}\right), 0.86\left(\mathrm{~s}, 6 \mathrm{H}, \mathrm{CMe}_{2}\right) .{ }^{13} \mathrm{C}\left\{{ }^{1} \mathrm{H}\right\} \mathrm{NMR}\left(\mathrm{CDCl}_{3}\right.$, 75.41 MHz, $\delta): 172.26,135.74,117.02,71.78,45.13,36.38,35.01,27.19,25.59$. IR $\left(\mathrm{CH}_{2} \mathrm{Cl}_{2}\right.$ soln, $\left.v_{\mathrm{C}=\mathrm{N}}, \mathrm{cm}^{-1}\right): 1669.3$.

\footnotetext{
${ }^{1}$ Sanford, M. S.; Love, J. A.; Grubbs, R. H. Organometallics 2001, 20, 5314-5318.

${ }^{2}$ Wakamatsu, H.; Blechert, S. Angew. Chem. Int. Ed. 2002, 41, 2403-2405.

${ }^{3}$ Grela, K.; Harutyunyan, S.; Michrowska, A. Angew. Chem. Int. Ed. 2002, 41, 4038-4040.

${ }^{4}$ Bender, C. F.; Widenhoefer, R. A. J Am. Chem. Soc. 2005, 127, 1070-1071.

${ }^{5}$ Pangborn, A. B.; Giardello, M. A.; Grubbs, R. H.; Rosen, R. K.; Timmers, F. J. Organometallics 1996, 15, 518-1520.
} 
General procedure for the synthesis of catalysts $\mathbf{9 a - b}$

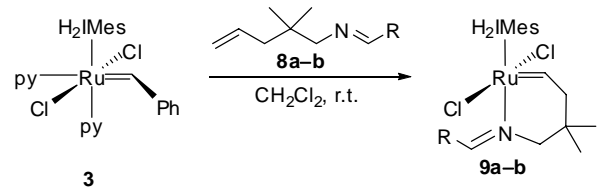

In the glove box, a Schlenk flask was charged with 3 and $\mathrm{CH}_{2} \mathrm{Cl}_{2}$. The corresponding imine was then added via syringe and the reaction stirred at r.t. for $30 \mathrm{~min}$. The volatiles were removed under vacuum, the residue redissolved in $\mathrm{C}_{6} \mathrm{H}_{6}(2 \mathrm{~mL})$, and precipitated with pentane $(20 \mathrm{~mL})$, cooling to $-5{ }^{\circ} \mathrm{C}$. The solid was collected, washed with pentane $(3 \times 5 \mathrm{~mL})$ and dried under vacuum to give the imine-substituted ruthenium compounds in good yields. Any modifications are described below for each reaction.

$\mathrm{R}=\mathrm{CH}_{2} \mathrm{Ph}(\mathbf{9 a})$

Ru complex 3 (196 mg, $0.270 \mathrm{mmol}$ ), imine 8a (67.9 mg, $0.337 \mathrm{mmol}$ ) and $\mathrm{CH}_{2} \mathrm{Cl}_{2}$ (5 mL) gave 9a (151 mg, $0.135 \mathrm{mmol})$ as a light green solid. Yield: $84 \%$. ${ }^{1} \mathrm{H} \mathrm{NMR}\left(\mathrm{CD}_{2} \mathrm{Cl}_{2}, 299.82 \mathrm{MHz}\right.$, $\delta): 18.71\left(\mathrm{t}, J=5.7 \mathrm{~Hz}, 1 \mathrm{H}, \mathrm{Ru}=\mathrm{CHCH}_{2}\right), 8.39(\mathrm{~s}, 1 \mathrm{H}, \mathrm{CH}=\mathrm{N}), 7.31(\mathrm{t}, J=7.5 \mathrm{~Hz}, 1 \mathrm{H}, \mathrm{Bn})$, 7.19 (d, $J=7.2 \mathrm{~Hz}, 2 \mathrm{H}, \mathrm{Bn}$ ), 7.06 (t, $J=7.8 \mathrm{~Hz}, 2 \mathrm{H}, \mathrm{Bn}$ ), 7.06 (s, $4 \mathrm{H}, \mathrm{Mes}$ ), 3.95 (s, $2 \mathrm{H}$, $\mathrm{CMe}_{2} \mathrm{CH}_{2} \mathrm{~N}$ ), $3.88\left(\mathrm{~s}, 4 \mathrm{H}, \mathrm{NCH}_{2} \mathrm{CH}_{2} \mathrm{~N}\right.$ ), $2.78\left(\mathrm{~d}, J=5.4 \mathrm{~Hz}, 2 \mathrm{H}, \mathrm{Ru}=\mathrm{CHCH}_{2} \mathrm{CMe}_{2}\right), 2.44(\mathrm{~s}, 12$ $\mathrm{H}, \mathrm{Mes}-\mathrm{CH}_{3}$ ), 2.41 (s, $\left.6 \mathrm{H}, \mathrm{Mes}-\mathrm{CH}_{3}\right), 0.73$ (s, $\left.6 \mathrm{H}, \mathrm{CMe}\right)_{2} .{ }^{13} \mathrm{C}\left\{{ }^{1} \mathrm{H}\right\} \mathrm{NMR}\left(\mathrm{CD}_{2} \mathrm{Cl}_{2}, 75.42 \mathrm{MHz}\right.$, $\delta): 341.41\left(\mathrm{Ru}=C \mathrm{CHCH}_{2}\right), 218.02\left(\mathrm{Ru}-\mathrm{C}(\mathrm{N})_{2}\right), 170.04(\mathrm{Ru}-\mathrm{N}=C), 138.87,137.29,134.62$, 130.96, 130.31, 130.11, 128.99, 77.35, 66.70, 51.91, 34.29, 26.35, 21.45, 19.41. IR $\left(\mathrm{CH}_{2} \mathrm{Cl}_{2}\right.$ soln, $\left.v_{\mathrm{C}=\mathrm{N}}, \mathrm{cm}^{-1}\right)$ : 1623.7. HRMS-FAB (m/z): [M] ${ }^{+}$calcd for $\mathrm{C}_{34} \mathrm{H}_{43} \mathrm{Cl}_{2} \mathrm{~N}_{3} \mathrm{Ru}$, 665.1878; found, 665.1855.

$\mathrm{R}=\mathrm{CMe}_{3}(\mathbf{9 b})$

Ru complex 3 (132 mg, $0.182 \mathrm{mmol}$ ), imine $8 \mathbf{b}$ (41.6 mg, $0.229 \mathrm{mmol}$ ) and $\mathrm{CH}_{2} \mathrm{Cl}_{2}$ (5 mL) gave 9b (92.0 mg, $0.142 \mathrm{mmol})$ as a light green solid. Yield: $78 \%{ }^{1} \mathrm{H} \mathrm{NMR}\left(\mathrm{CD}_{2} \mathrm{Cl}_{2}, 299.82 \mathrm{MHz}\right.$, $\delta$ ): 18.54 (t, $J=6.0 \mathrm{~Hz}, 1 \mathrm{H}, \mathrm{Ru}=\mathrm{CHCH}_{2}$ ), 7.45 (t, $J=1.5 \mathrm{~Hz}, 1 \mathrm{H}, \mathrm{CH}=\mathrm{N}$ ), 7.00 (br s, $4 \mathrm{H}$, Mes), 3.90 (br s, $4 \mathrm{H}, \mathrm{NCH}_{2} \mathrm{CH}_{2} \mathrm{~N}$ ), 3.45 (d, $J=1.8 \mathrm{~Hz}, 2 \mathrm{H}, \mathrm{CMe}_{2} \mathrm{CH}_{2} \mathrm{~N}$ ), 2.81 (d, $J=6.3 \mathrm{~Hz}, 2$ $\mathrm{H}, \mathrm{Ru}=\mathrm{CHCH}_{2} \mathrm{CMe}_{2}$ ), 2.42 (br s, $12 \mathrm{H}, \mathrm{Mes}-\mathrm{CH}_{3}$ ), 2.35 (s, $6 \mathrm{H}, \mathrm{Mes}-\mathrm{CH}_{3}$ ), 0.95 (s, $9 \mathrm{H}$, $\left.\mathrm{N}=\mathrm{CHCMe}_{3}\right), \quad 0.70$ (s, $\left.6 \mathrm{H}, \mathrm{CMe} e_{2}\right) .{ }^{13} \mathrm{C}\left\{{ }^{1} \mathrm{H}\right\} \quad \mathrm{NMR}\left(\mathrm{CD}_{2} \mathrm{Cl}_{2}, 125.71 \mathrm{MHz}, \delta\right): 343.35$ $\left(\mathrm{Ru}=\mathrm{CHCH}_{2}\right), 218.91\left(\mathrm{Ru}-\mathrm{C}(\mathrm{N})_{2}\right), 180.80(\mathrm{Ru}-\mathrm{N}=\mathrm{C}), 138.74,137.48,130.02,76.96,66.43$, 51.60, 37.02, 34.61, 26.53, 26.45, 21.36, 19.52 (br). IR $\left(\mathrm{CH}_{2} \mathrm{Cl}_{2}\right.$ soln, $\left.v_{\mathrm{C}=\mathrm{N}}, \mathrm{cm}^{-1}\right)$ : 1635.6. HRMS-FAB (m/z): [M] $]^{+}$calcd for $\mathrm{C}_{32} \mathrm{H}_{47} \mathrm{Cl}_{2} \mathrm{~N}_{3} \mathrm{Ru}, 645.2191$; found, 645.2204. 
General procedure for the synthesis of imines $\mathrm{CH}_{2}=\mathrm{CHCH}_{2} \mathrm{CMe}_{2} \mathrm{CH}=\mathrm{NR}(\mathbf{1 4 a}-\mathbf{e})$

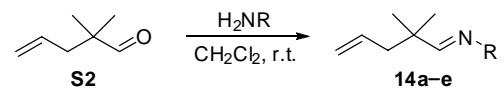

The condensation of 2,2-dimethyl-4-pentenal (S2) with various primary amines was carried out in $\mathrm{CH}_{2} \mathrm{Cl}_{2}$ over activated $4 \AA$ molecular sieves at r.t. for $12 \mathrm{~h}$. The sieves were removed by filtration and the solution concentrated under vacuum to give the desired imines.

$\mathrm{R}=\mathrm{Ph},(\mathbf{1 4 a})$

Aldehyde S2 (1.00 mL, $0.825 \mathrm{~g}, 7.35 \mathrm{mmol})$ and aniline $(0.67 \mathrm{~mL}, 0.674 \mathrm{~g}, 7.35 \mathrm{mmol})$ in $\mathrm{CH}_{2} \mathrm{Cl}_{2}(15 \mathrm{~mL})$ gave 14a $(1.094 \mathrm{~g}, 5.84 \mathrm{mmol})$ as a clear liquid. Yield: $79 \%$. ${ }^{1} \mathrm{H} \mathrm{NMR}$ $\left(\mathrm{CDCl}_{3}, 300.09 \mathrm{MHz}, \delta\right): 7.69(\mathrm{~s}, 1 \mathrm{H}, \mathrm{CH}=\mathrm{N})$, 7.35-6.95 (m, $\left.5 \mathrm{H}, \mathrm{Ph}\right)$, 5.92-5.76 (m, $1 \mathrm{H}$, $\mathrm{CH}_{2}=\mathrm{CHCH}_{2}$ ), 5.12-5.04 (m, $\left.2 \mathrm{H}, \mathrm{CH}_{2}=\mathrm{CH}\right), 2.29$ (dt, $J=7.5,1.2 \mathrm{~Hz}, 2 \mathrm{H},=\mathrm{CHCH}_{2} \mathrm{CMe}_{2}$ ), 1.18 (s, $\left.6 \mathrm{H}, \mathrm{CMe})_{2}\right){ }^{13} \mathrm{C}\left\{{ }^{1} \mathrm{H}\right\} \mathrm{NMR}\left(\mathrm{CDCl}_{3}, 75.46 \mathrm{MHz}, \delta\right): 172.78,152.84,134.58,129.12$, 125.36, 120.74, 117.98, 44.87, 39.94, 24.63. IR $\left(\mathrm{CH}_{2} \mathrm{Cl}_{2}\right.$ soln, $\left.v_{\mathrm{C}=\mathrm{N}}, \mathrm{cm}^{-1}\right): 1648.0$.

$\mathrm{R}={ }^{i} \operatorname{Pr}(\mathbf{1 4 b})$

Aldehyde S2 (1.00 mL, $0.825 \mathrm{~g}, 7.35 \mathrm{mmol})$ and isopropyl amine (1.25 mL, $0.867 \mathrm{~g}, 14.7 \mathrm{mmol})$ in $\mathrm{CH}_{2} \mathrm{Cl}_{2}(15 \mathrm{~mL})$ gave $14 \mathbf{b}(0.666 \mathrm{~g}, 4.34 \mathrm{mmol})$ as a clear liquid. Yield: $59 \%$. ${ }^{1} \mathrm{H} \mathrm{NMR}$ $\left(\mathrm{CDCl}_{3}, 300.08 \mathrm{MHz}, \delta\right): 7.49(\mathrm{~s}, 1 \mathrm{H}, \mathrm{CH}=\mathrm{N}), 5.82-5.64\left(\mathrm{~m}, 1 \mathrm{H}, \mathrm{CH}_{2}=\mathrm{CHCH}_{2}\right), 5.06-4.92(\mathrm{~m}$, $2 \mathrm{H}, \mathrm{CH}_{2}=\mathrm{CH}$ ), 3.24 (sept., $J=6.3 \mathrm{~Hz}, 1 \mathrm{H}, \mathrm{NCHMe}_{2}$ ), 2.13 (d, $J=7.2 \mathrm{~Hz}, 2 \mathrm{H}$, $\left.=\mathrm{CHCH}_{2} \mathrm{CMe}_{2}\right), 1.11\left(\mathrm{~d}, J=6.6 \mathrm{~Hz}, 6 \mathrm{H}, \mathrm{NCHMe}\right.$ ), $1.02\left(\mathrm{~s}, 6 \mathrm{H}, \mathrm{CMe} e_{2}\right){ }^{13} \mathrm{C}\left\{{ }^{1} \mathrm{H}\right\} \mathrm{NMR}$ $\left(\mathrm{CDCl}_{3}, 75.46 \mathrm{MHz}, \delta\right): 168.35,134.97,117.41,61.69,44.99$, 38.62, 24.92, 24.35. IR $\left(\mathrm{CH}_{2} \mathrm{Cl}_{2}\right.$ soln, $\left.v_{\mathrm{C}=\mathrm{N}}, \mathrm{cm}^{-1}\right): 1661.4$.

\section{$\mathrm{R}=\mathrm{Cy}(\mathbf{1 4 c})$}

Aldehyde S2 (1.00 mL, $0.825 \mathrm{~g}, 7.35 \mathrm{mmol})$ and cyclohexyl amine (0.88 mL, $0.762 \mathrm{~g}, 7.69$ mmol) in $\mathrm{CH}_{2} \mathrm{Cl}_{2}(15 \mathrm{~mL})$ gave $14 \mathrm{c}(0.730 \mathrm{~g}, 3.77 \mathrm{mmol})$ as a clear liquid. Yield: $51 \%$. ${ }^{1} \mathrm{H}$ NMR $\left(\mathrm{CDCl}_{3}, 300.08 \mathrm{MHz}, \delta\right): 7.50(\mathrm{~s}, 1 \mathrm{H}, \mathrm{CH}=\mathrm{N}), 5.82-5.66\left(\mathrm{~m}, 1 \mathrm{H}, \mathrm{CH}_{2}=\mathrm{CHCH}_{2}\right)$, 5.04-4.94 (m, $\left.2 \mathrm{H}, \mathrm{CH}_{2}=\mathrm{CH}\right), 2.89$ (tt, $J=10.5,4.2 \mathrm{~Hz}, 1 \mathrm{H}, \mathrm{NCH}-\mathrm{Cy}$ ), 2.13 (dt, $J=7.5 \mathrm{~Hz}$, $\left.\left.0.9 \mathrm{~Hz}, 2 \mathrm{H},=\mathrm{CHCH}_{2} \mathrm{CMe}_{2}\right), 1.82-1.10(\mathrm{~m}, 10 \mathrm{H}, \mathrm{Cy}), 1.01(\mathrm{~s}, 6 \mathrm{H}, \mathrm{CMe})_{2}\right){ }^{13} \mathrm{C}\left\{{ }^{1} \mathrm{H}\right\} \mathrm{NMR}$ $\left(\mathrm{CDCl}_{3}, 75.46 \mathrm{MHz}, \delta\right):$ 168.73, 135.03, 117.37, 70.04, 45.00, 38.69, 34.60, 25.81, 25.10, 24.94. IR $\left(\mathrm{CH}_{2} \mathrm{Cl}_{2}\right.$ soln, $\left.v_{\mathrm{C}=\mathrm{N}}, \mathrm{cm}^{-1}\right): 1662.7$.

$\mathrm{R}={ }^{t} \mathrm{Bu}(\mathbf{1 4 d})$

Aldehyde S2 (1.00 mL, $0.825 \mathrm{~g}, 7.35 \mathrm{mmol})$ and t-butyl amine (0.93 mL, $0.647 \mathrm{~g}, 8.85 \mathrm{mmol})$ in $\mathrm{CH}_{2} \mathrm{Cl}_{2}(15 \mathrm{~mL})$ gave 14d $(0.555 \mathrm{~g}, 3.32 \mathrm{mmol})$ as a clear liquid. Yield: $45 \%$. ${ }^{1} \mathrm{H} \mathrm{NMR}$ $\left(\mathrm{CDCl}_{3}, 300.08 \mathrm{MHz}, \delta\right): 7.43(\mathrm{~s}, 1 \mathrm{H}, \mathrm{CH}=\mathrm{N}), 5.82-5.66\left(\mathrm{~m}, 1 \mathrm{H}, \mathrm{CH}_{2}=\mathrm{CHCH}_{2}\right), 5.03-4.94(\mathrm{~m}$, $2 \mathrm{H}, \mathrm{CH}_{2}=\mathrm{CH}$ ), 2.14 (dt, $J=7.5,1.2 \mathrm{~Hz}, 2 \mathrm{H},=\mathrm{CHCH}_{2} \mathrm{CMe}_{2}$ ), 1.13 (s, $9 \mathrm{H}, \mathrm{NCMe}_{3}$ ), 1.01 (s, 6 $\left.\mathrm{H}, \mathrm{CMe} e_{2}\right) .{ }^{13} \mathrm{C}\left\{{ }^{1} \mathrm{H}\right\} \mathrm{NMR}\left(\mathrm{CDCl}_{3}, 75.46 \mathrm{MHz}, \delta\right): 164.63,135.31,117.19,56.42,45.09,38.68$, 29.97, 24.92. IR $\left(\mathrm{CH}_{2} \mathrm{Cl}_{2}\right.$ soln, $\left.v_{\mathrm{C}=\mathrm{N}}, \mathrm{cm}^{-1}\right): 1665.9$. 
$\mathrm{R}=\mathrm{Me}(\mathbf{1 4 e})$

Aldehyde S2 (1.00 mL, $0.825 \mathrm{~g}, 7.35 \mathrm{mmol}$ ) and methyl amine (2.0 M THF, $6.00 \mathrm{~mL}, 12.0$ mmol) in $\mathrm{CH}_{2} \mathrm{Cl}_{2}(15 \mathrm{~mL})$ gave $14 \mathrm{e}(0.631 \mathrm{~g}, 5.04 \mathrm{mmol})$ as a clear liquid. Yield: $69 \%$. ${ }^{1} \mathrm{H}$ NMR $\left(\mathrm{CDCl}_{3}, 300.09 \mathrm{MHz}, \delta\right): 7.49$ (q, $\left.J=1.5 \mathrm{~Hz}, 1 \mathrm{H}, \mathrm{CH}=\mathrm{N}\right), 5.80-5.64(\mathrm{~m}, 1 \mathrm{H}$, $\mathrm{CH}_{2}=\mathrm{CHCH}_{2}$ ), 5.04-4.94 (m, $2 \mathrm{H}, \mathrm{CH}_{2}=\mathrm{CH}$ ), 3.23 (d, $J=1.5 \mathrm{~Hz}, 3 \mathrm{H}, \mathrm{NMe}$ ), 2.12 (dt, $J=7$. 2, $\left.1.2 \mathrm{~Hz}, 2 \mathrm{H},=\mathrm{CHCH}_{2} \mathrm{CMe}_{2}\right), 1.01\left(\mathrm{~s}, 6 \mathrm{H}, \mathrm{CMe} e_{2}\right) .{ }^{13} \mathrm{C}\left\{{ }^{1} \mathrm{H}\right\} \mathrm{NMR}\left(\mathrm{CDCl}_{3}, 75.46 \mathrm{MHz}, \delta\right)$ : 172.72, 134.74, 117.59, 48.08, 44.79, 39.13, 24.63. IR $\left(\mathrm{CH}_{2} \mathrm{Cl}_{2}\right.$ soln, $\left.v_{\mathrm{C}=\mathrm{N}}, \mathrm{cm}^{-1}\right): 1670.9$.

General procedure for the synthesis of catalysts $\mathbf{1 5 a}-\mathbf{e}$

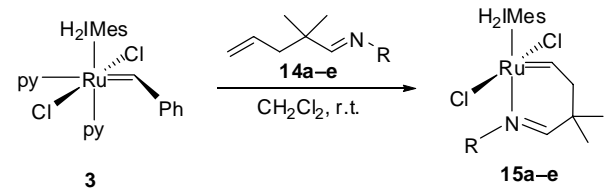

In the glove box, a Schlenk flask was charged with 3 and $\mathrm{CH}_{2} \mathrm{Cl}_{2}$. The corresponding imine was then added via syringe and the reaction stirred at r.t. for $30 \mathrm{~min}$. The volatiles were removed under vacuum, the residue redissolved in $\mathrm{C}_{6} \mathrm{H}_{6}(2 \mathrm{~mL})$, and precipitated with pentane $(20 \mathrm{~mL})$, cooling to $-5{ }^{\circ} \mathrm{C}$. The solid was collected, washed with pentane $(3 \times 5 \mathrm{~mL})$ and dried under vacuum to give the imine-substituted ruthenium compounds in good yields. Any modifications are described below for each reaction.

$\mathrm{R}=\mathrm{Ph}(\mathbf{1 5 a})$

Ru complex 3 (155 mg, $0.213 \mathrm{mmol})$, imine 14a (59.8 mg, $0.319 \mathrm{mmol})$ and $\mathrm{CH}_{2} \mathrm{Cl}_{2}(5 \mathrm{~mL})$ gave 15a (116 mg, $0.177 \mathrm{mmol})$ as a light green solid. Yield: $83 \%$. ${ }^{1} \mathrm{H} \mathrm{NMR}\left(\mathrm{CD}_{2} \mathrm{Cl}_{2}, 299.87\right.$ $\mathrm{MHz}, \delta): 18.80\left(\mathrm{t}, J=5.4 \mathrm{~Hz}, 1 \mathrm{H}, \mathrm{Ru}=\mathrm{CHCH}_{2}\right), 7.64$ (s, $\left.1 \mathrm{H}, \mathrm{CH}=\mathrm{N}\right), 7.2-6.9(\mathrm{~m}, 9 \mathrm{H}, \mathrm{Ph}$ and Mes), 4.01 (s, $4 \mathrm{H}, \mathrm{NCH}_{2} \mathrm{CH}_{2} \mathrm{~N}$ ), 3.02 (d, $J=5.4 \mathrm{~Hz}, 2 \mathrm{H}, \mathrm{Ru}=\mathrm{CHCH}_{2} \mathrm{CMe}_{2}$ ), 2.5-2.3 (m, $18 \mathrm{H}$, Mes- $\left.\mathrm{CH}_{3}\right), 1.07$ (s, $\left.\left.6 \mathrm{H}, \mathrm{CMe}\right)_{2}\right){ }^{13} \mathrm{C}\left\{{ }^{1} \mathrm{H}\right\}$ NMR $\left(\mathrm{CD}_{2} \mathrm{Cl}_{2}, 125.71 \mathrm{MHz}, \delta\right): 345.10\left(\mathrm{Ru}=\mathrm{CHCH}_{2}\right)$, $218.03\left(\mathrm{Ru}-\mathrm{C}(\mathrm{N})_{2}\right), 176.96(\mathrm{Ru}-\mathrm{N}=C), 149.63,138.81,129.82,129.40,127.12,122.48,64.30$, 51.82, 42.69, 26.89, 21.46, 19.28. IR $\left(\mathrm{CH}_{2} \mathrm{Cl}_{2}\right.$ soln, $\left.v_{\mathrm{C}=\mathrm{N}}, \mathrm{cm}^{-1}\right)$ : 1634.3. HRMS-FAB (m/z): $[\mathrm{M}]^{+}$calcd for $\mathrm{C}_{33} \mathrm{H}_{41} \mathrm{Cl}_{2} \mathrm{~N}_{3} \mathrm{Ru}$, 651.1722; found, 651.1726. Anal. Calcd for $\mathrm{C}_{33} \mathrm{H}_{41} \mathrm{Cl}_{2} \mathrm{~N}_{3} \mathrm{Ru}$ : C, 60.82; H, 6.34; N, 6.45. Found: C, 60.72; H, 6.38; N, 6.48.

$\mathrm{R}={ }^{i} \operatorname{Pr}(\mathbf{1 5 b})$

Ru complex 3 (239 mg, $0.328 \mathrm{mmol}$ ), imine 14b (75.7 mg, $0.493 \mathrm{mmol})$ and $\mathrm{CH}_{2} \mathrm{Cl}_{2}(5 \mathrm{~mL})$ gave 15b (162 mg, $0.262 \mathrm{mmol})$ as a light green solid. Yield: $80 \%$. ${ }^{1} \mathrm{H} \mathrm{NMR}\left(\mathrm{CD}_{2} \mathrm{Cl}_{2}, 299.82\right.$ $\mathrm{MHz}, \delta$ ): 18.58 (t, $\left.J=5.4 \mathrm{~Hz}, 1 \mathrm{H}, \mathrm{Ru}=\mathrm{CHCH}_{2}\right), 7.41$ (d, $\left.J=1.5 \mathrm{~Hz}, 1 \mathrm{H}, \mathrm{CH}=\mathrm{N}\right), 6.99$ (s, $4 \mathrm{H}$, Mes), 4.02 (s, $4 \mathrm{H}, \mathrm{NCH}_{2} \mathrm{CH}_{2} \mathrm{~N}$ ), 3.32 (sept.d, $J=6.6,1.5 \mathrm{~Hz}, 1 \mathrm{H}, \mathrm{NCHMe}$ ), 2.96 (d, $J=5.4$ $\mathrm{Hz}, 2 \mathrm{H}, \mathrm{Ru}=\mathrm{CHCH}_{2} \mathrm{CMe}_{2}$ ), 2.42 (br s, $12 \mathrm{H}, \mathrm{Mes}-\mathrm{CH}_{3}$ ), 2.34 (s, $6 \mathrm{H}, \mathrm{Mes}-\mathrm{CH}_{3}$ ), 0.92 (s, $6 \mathrm{H}$, $\mathrm{CMe}_{2}$ ), 0.90 (d, $\left.J=6.9 \mathrm{~Hz}, 6 \mathrm{H}, \mathrm{NCHMe} 2\right) .{ }^{13} \mathrm{C}\left\{{ }^{1} \mathrm{H}\right\} \mathrm{NMR}\left(\mathrm{CD}_{2} \mathrm{Cl}_{2}, 125.71 \mathrm{MHz}, \delta\right): 345.17$ $\left(\mathrm{Ru}=\mathrm{CHCH}_{2}\right), 219.54\left(\mathrm{Ru}-\mathrm{C}(\mathrm{N})_{2}\right), 173.68(\mathrm{Ru}-\mathrm{N}=\mathrm{C}), 138.91,129.74,64.21,60.78,51.60$, 
42.51, 26.96, 22.47, 21.36, 19.36 (br). IR $\left(\mathrm{CH}_{2} \mathrm{Cl}_{2}\right.$ soln, $\left.v_{\mathrm{C}=\mathrm{N}}, \mathrm{cm}^{-1}\right)$ : 1642.6. HRMS-FAB (m/z): $[\mathrm{M}]^{+}$calcd for $\mathrm{C}_{30} \mathrm{H}_{43} \mathrm{Cl}_{2} \mathrm{~N}_{3} \mathrm{Ru}, 617.1878$; found, 617.1853.

$\mathrm{R}=\mathrm{Cy}(\mathbf{1 5 c})$

Ru complex 3 (192 mg, $0.263 \mathrm{mmol}$ ), imine 14c (74.0 mg, $0.382 \mathrm{mmol})$ and $\mathrm{CH}_{2} \mathrm{Cl}_{2}(5 \mathrm{~mL})$ gave 15c (146 mg, $0.222 \mathrm{mmol}$ ) as a light green solid. Yield: $84 \%$. ${ }^{1} \mathrm{H} \mathrm{NMR}\left(\mathrm{CD}_{2} \mathrm{Cl}_{2}, 299.87\right.$ MHz, $\delta$ ): 18.56 (t, $J=5.4 \mathrm{~Hz}, 1 \mathrm{H}, \mathrm{Ru}=\mathrm{CHCH}_{2}$ ), 7.41 (d, $J=0.9 \mathrm{~Hz}, 1 \mathrm{H}, \mathrm{CH}=\mathrm{N}$ ), 7.00 (br s, 4 H, Mes), 4.00 (br s, $4 \mathrm{H}, \mathrm{NCH}_{2} \mathrm{CH}_{2} \mathrm{~N}$ ), 2.96 (d, $J=5.7 \mathrm{~Hz}, 2 \mathrm{H}, \mathrm{Ru}=\mathrm{CHCH}_{2} \mathrm{CMe}_{2}$ ), 2.7-2.2 (br m, $12 \mathrm{H}$, Mes- $\mathrm{CH}_{3}$ ), 2.34 (s, $6 \mathrm{H}$, Mes- $\mathrm{CH}_{3}$ ), 1.7-0.8 (m, $11 \mathrm{H}, \mathrm{Cy}$ ), 0.91 (s, $6 \mathrm{H}, \mathrm{CMe}_{2}$ ). ${ }^{13} \mathrm{C}\left\{{ }^{1} \mathrm{H}\right\}$ NMR $\left(\mathrm{CD}_{2} \mathrm{Cl}_{2}, 125.71 \mathrm{MHz}, \delta\right): 345.00\left(\mathrm{Ru}=\mathrm{CHCH}_{2}\right), 219.49\left(\mathrm{Ru}-\mathrm{C}(\mathrm{N})_{2}\right), 173.76$ $(\mathrm{Ru}-\mathrm{N}=C), 138.76,129.76,69.99,64.07,51.63,42.23,33.47,26.90,26.10,25.49,21.39,20.00$ (br), 18.78 (br). IR $\left(\mathrm{CH}_{2} \mathrm{Cl}_{2}\right.$ soln, $\left.v_{\mathrm{C}=\mathrm{N}}, \mathrm{cm}^{-1}\right)$ : 1641.1. HRMS-FAB (m/z): [M] $]^{+}$calcd for $\mathrm{C}_{33} \mathrm{H}_{47} \mathrm{Cl}_{2} \mathrm{~N}_{3} \mathrm{Ru}$, 657.2191; found, 657.2163.

$\mathrm{R}={ }^{t} \mathrm{Bu}(\mathbf{1 5 d})$

Ru complex 3 (188 mg, $0.258 \mathrm{mmol}$ ), imine 14d (56.0 mg, $0.335 \mathrm{mmol}$ ) and $\mathrm{CH}_{2} \mathrm{Cl}_{2}(5 \mathrm{~mL})$ gave 15d (90.8 mg, $0.143 \mathrm{mmol})$ as a light green solid. Yield: $56 \%$. ${ }^{1} \mathrm{H} \mathrm{NMR}\left(\mathrm{CD}_{2} \mathrm{Cl}_{2}, 299.82\right.$ $\mathrm{MHz}, \delta$ ): 18.37 (t, $J=5.7 \mathrm{~Hz}, 1 \mathrm{H}, \mathrm{Ru}=\mathrm{CHCH}_{2}$ ), 7.43 (s, $1 \mathrm{H}, \mathrm{CH}=\mathrm{N}$ ), 7.04-6.94 (m, $4 \mathrm{H}, \mathrm{Mes}$ ), 4.10-3.96 (m, $4 \mathrm{H}, \mathrm{NCH}_{2} \mathrm{CH}_{2} \mathrm{~N}$ ), 3.08 (d, $J=5.4 \mathrm{~Hz}, 2 \mathrm{H}, \mathrm{Ru}=\mathrm{CHCH}_{2} \mathrm{CMe}_{2}$ ), 2.59 (br s, $6 \mathrm{H}$, Mes- $\mathrm{CH}_{3}$ ), 2.34 (s, $6 \mathrm{H}$, Mes- $\mathrm{CH}_{3}$ ), 2.26 (br s, $6 \mathrm{H}$, Mes- $\mathrm{CH}_{3}$ ), 1.01 (s, $9 \mathrm{H}, \mathrm{NCMe}_{3}$ ), 0.92 (s, 6 $\mathrm{H}, \mathrm{CMe} 2) .{ }^{13} \mathrm{C}\left\{{ }^{1} \mathrm{H}\right\}$ NMR $\left(\mathrm{CD}_{2} \mathrm{Cl}_{2}, 125.71 \mathrm{MHz}, \delta\right): 345.22\left(\mathrm{Ru}=\mathrm{CHCH}_{2}\right), 219.82\left(\mathrm{Ru}-\mathrm{C}(\mathrm{N})_{2}\right)$, $172.97(\mathrm{Ru}-\mathrm{N}=C), 139.83,139.13,138.55,137.92,136.09,129.83,129.74,64.05,63.66,51.75$, 51.27, 43.02, 28.89, 26.77, 21.37, 20.21, 18.58. IR $\left(\mathrm{CH}_{2} \mathrm{Cl}_{2}\right.$ soln, $\left.v_{\mathrm{C}=\mathrm{N}}, \mathrm{cm}^{-1}\right)$ : 1638.6. HRMS-FAB (m/z): [M] ${ }^{+}$calcd for $\mathrm{C}_{31} \mathrm{H}_{45} \mathrm{Cl}_{2} \mathrm{~N}_{3} \mathrm{Ru}, 631.2035$; found, 631.2031.

$\mathrm{R}=\mathrm{Me}(\mathbf{1 5 e})$

Ru complex 3 (143 mg, $0.196 \mathrm{mmol}$ ), imine 14e (30.4 mg, $0.242 \mathrm{mmol}$ ) and $\mathrm{CH}_{2} \mathrm{Cl}_{2}(5 \mathrm{~mL})$ gave 15e $(92.5 \mathrm{mg}, 0.164 \mathrm{mmol})$ as a green-brown solid. Yield: $84 \%$. ${ }^{1} \mathrm{H}$ NMR $\left(\mathrm{CD}_{2} \mathrm{Cl}_{2}\right.$, $299.87 \mathrm{MHz}, \delta$ ): 18.80 (t, $J=5.1 \mathrm{~Hz}, 1 \mathrm{H}, \mathrm{Ru}=\mathrm{CHCH}_{2}$ ), $7.42(\mathrm{~m}, 1 \mathrm{H}, \mathrm{CH}=\mathrm{N}), 7.00$ (br s, $4 \mathrm{H}$, Mes), 4.05 (s, $4 \mathrm{H}, \mathrm{NCH}_{2} \mathrm{CH}_{2} \mathrm{~N}$ ), $2.73(\mathrm{~d}, J=1.2 \mathrm{~Hz}, 1 \mathrm{H}, \mathrm{NMe}$ ), 2.69 (d, $J=5.1 \mathrm{~Hz}, 2 \mathrm{H}$, $\mathrm{Ru}=\mathrm{CHCH}_{2} \mathrm{CMe}_{2}$ ), 2.41 (s, $12 \mathrm{H}$, Mes- $\mathrm{CH}_{3}$ ), 2.34 (s, $6 \mathrm{H}, \mathrm{Mes}-\mathrm{CH}_{3}$ ), 0.93 (s, $6 \mathrm{H}, \mathrm{CMe}_{2}$ ). ${ }^{13} \mathrm{C}\left\{{ }^{1} \mathrm{H}\right\}$ NMR $\left(\mathrm{CD}_{2} \mathrm{Cl}_{2}, 125.71 \mathrm{MHz}, \delta\right): 342.54\left(\mathrm{Ru}=\mathrm{CHCH}_{2}\right), 218.93\left(\mathrm{Ru}-\mathrm{C}(\mathrm{N})_{2}\right), 175.29$ $(\mathrm{Ru}-\mathrm{N}=C), 139.04,138.87,136.52,129.61,64.46,51.85,46.76,41.83,26.88,21.37,19.56 . \mathrm{IR}$ $\left(\mathrm{CH}_{2} \mathrm{Cl}_{2}\right.$ soln, $\left.v_{\mathrm{C}=\mathrm{N}}, \mathrm{cm}^{-1}\right)$ : 1635.4. HRMS-FAB (m/z): [M] $]^{+}$calcd for $\mathrm{C}_{28} \mathrm{H}_{39} \mathrm{Cl}_{2} \mathrm{~N}_{3} \mathrm{Ru}$, 589.1565; found, 589.1560. 
General procedure for the synthesis of imines $\mathrm{CH}_{2}=\mathrm{CHCH}_{2} \mathrm{CMe}_{2} \mathrm{CH}=\mathrm{NCH}_{2} \mathrm{CH}_{2} \mathrm{XMe}(\mathbf{1 6 a}-\mathbf{1 6} \mathbf{c})$

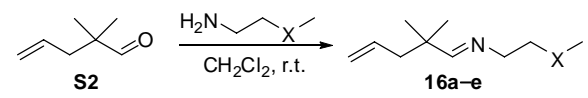

The condensation of 2,2-dimethyl-4-pentenal (S2) with various primary amines was carried out in $\mathrm{CH}_{2} \mathrm{Cl}_{2}$ over activated $4 \AA$ molecular sieves at r.t. for $12 \mathrm{~h}$. The sieves were removed by filtration and the solution concentrated under vacuum to give the desired imines.

$\mathrm{X}=\mathrm{CH}_{2}$ (16a)

Aldehyde S2 (1.00 mL, 0.825 g, $7.35 \mathrm{mmol})$ and n-butylamine (1.09 mL, $0.806 \mathrm{~g}, 11.0 \mathrm{mmol})$ in $\mathrm{CH}_{2} \mathrm{Cl}_{2}(15 \mathrm{~mL})$ gave 16a $(0.705 \mathrm{~g}, 4.22 \mathrm{mmol})$ as a clear liquid. Yield: $57 \%$. ${ }^{1} \mathrm{H} \mathrm{NMR}$ $\left(\mathrm{CDCl}_{3}, 300.09 \mathrm{MHz}, \delta\right): 7.47(\mathrm{t}, J=1.2 \mathrm{~Hz}, 1 \mathrm{H}, \mathrm{CH}=\mathrm{N}), 5.82-5.66\left(\mathrm{~m}, 1 \mathrm{H}, \mathrm{CH}_{2}=\mathrm{CHCH}_{2}\right)$, 5.06-4.94 (m, $2 \mathrm{H}, \mathrm{CH}_{2}=\mathrm{CH}$ ), 3.36 (td, $J=7.2,1.2 \mathrm{~Hz}, 2 \mathrm{H}, \mathrm{CH}=\mathrm{NCH}_{2} \mathrm{CH}_{2}$ ), 2.14 (d, $J=7.5$ $\mathrm{Hz}, 2 \mathrm{H}$, $=\mathrm{CHCH}_{2} \mathrm{CMe}_{2}$ ), 1.54 (quint., $J=7.5 \mathrm{~Hz}, 2 \mathrm{H}, \mathrm{NCH}_{2} \mathrm{CH}_{2} \mathrm{CH}_{2}$ ), 1.27 (sext., $J=7.8 \mathrm{~Hz}$, $\mathrm{CH}_{2} \mathrm{CH}_{2} \mathrm{Me}$ ), 1.03 (s, $\left.6 \mathrm{H}, \mathrm{CMe} e_{2}\right), 0.89$ (t, $\left.J=7.2 \mathrm{~Hz}, 3 \mathrm{H}, \mathrm{Bu}-\mathrm{Me}\right) .{ }^{13} \mathrm{C}\left\{{ }^{1} \mathrm{H}\right\} \mathrm{NMR}\left(\mathrm{CDCl}_{3}\right.$, 75.46 MHz, $\delta$ ): 171.12, 134.87, 117.51, 61.33, 44.92, 39.02, 33.15, 24.82, 20.40, 14.04. IR $\left(\mathrm{CH}_{2} \mathrm{Cl}_{2}\right.$ soln, $\left.v_{\mathrm{C}=\mathrm{N}}, \mathrm{cm}^{-1}\right): 1665.6$.

\section{$\mathrm{X}=\mathrm{O}(\mathbf{1 6 b})$}

Aldehyde S2 (1.00 mL, $0.825 \mathrm{~g}, 7.35 \mathrm{mmol})$ and 2-methoxyethylamine (0.77 mL, $0.662 \mathrm{~g}, 8.82$ mmol) in $\mathrm{CH}_{2} \mathrm{Cl}_{2}(15 \mathrm{~mL})$ gave $16 \mathbf{b}(0.946 \mathrm{~g}, 5.59 \mathrm{mmol})$ as a clear liquid. Yield: $76 \%$. ${ }^{1} \mathrm{H}$ NMR $\left(\mathrm{CDCl}_{3}, 299.82 \mathrm{MHz}, \delta\right): 7.53(\mathrm{~s}, 1 \mathrm{H}, \mathrm{CH}=\mathrm{N}), 5.83-5.67\left(\mathrm{~m}, 1 \mathrm{H}, \mathrm{CH}_{2}=\mathrm{CHCH}_{2}\right)$, 5.06-4.97 (m, $2 \mathrm{H}, \mathrm{CH}_{2}=\mathrm{CH}$ ), 3.55 (s, $4 \mathrm{H}, \mathrm{CH}=\mathrm{NCH}_{2} \mathrm{CH}_{2}$ ), 3.34 (s, $3 \mathrm{H}, \mathrm{OMe}$ ), 2.15 (d, J = 7.2 $\mathrm{Hz}, 2 \mathrm{H}$, =CHCH $\left.\mathrm{CMe}_{2}\right), 1.05\left(\mathrm{~s}, 6 \mathrm{H}, \mathrm{CMe}_{2}\right) .{ }^{13} \mathrm{C}\left\{{ }^{1} \mathrm{H}\right\} \mathrm{NMR}\left(\mathrm{CDCl}_{3}, 75.47 \mathrm{MHz}, \delta\right): 173.29$, 134.80, 117.50, 72.29, 60.98, 58.98, 44.84, 39.19. IR $\left(\mathrm{CH}_{2} \mathrm{Cl}_{2}\right.$ soln, $\left.v_{\mathrm{C}=\mathrm{N}}, \mathrm{cm}^{-1}\right): 1666.6$.

$\mathrm{X}=\mathrm{S}(\mathbf{1 6 c})$

Aldehyde S2 (1.00 mL, $0.825 \mathrm{~g}, 7.35 \mathrm{mmol})$ and 2-thiomethylethylamine (0.69 mL, $0.67 \mathrm{~g}, 7.37$ $\mathrm{mmol})$ in $\mathrm{CH}_{2} \mathrm{Cl}_{2}(15 \mathrm{~mL})$ gave 16c $(1.150 \mathrm{~g}, 6.20 \mathrm{mmol})$ as a pale yellow liquid. Yield: $84 \%$. ${ }^{1} \mathrm{H}$ NMR $\left(\mathrm{CDCl}_{3}, 299.82 \mathrm{MHz}, \delta\right): 7.53(\mathrm{t}, J=1.2 \mathrm{~Hz}, 1 \mathrm{H}, \mathrm{CH}=\mathrm{N}), 5.84-5.70(\mathrm{~m}, 1 \mathrm{H}$, $\mathrm{CH}_{2}=\mathrm{CHCH}_{2}$ ), 5.06-4.98 (m, $\left.2 \mathrm{H}, \mathrm{CH}_{2}=\mathrm{CH}\right), 3.58$ (td, $J=7.2,1.2 \mathrm{~Hz}, 2 \mathrm{H}, \mathrm{CH}=\mathrm{NCH}_{2} \mathrm{CH}_{2}$ ), 2.71 (t, $J=7.2 \mathrm{~Hz}, 2 \mathrm{H}, \mathrm{NCH}_{2} \mathrm{CH}_{2} \mathrm{SMe}$ ), 2.16 (td, $J=7.5,1.2 \mathrm{~Hz}, 2 \mathrm{H},=\mathrm{CHCH}_{2} \mathrm{CMe}_{2}$ ), 2.12 (s, $3 \mathrm{H}, \mathrm{CH}_{2} \mathrm{SMe}$ ), 1.05 (s, $\left.6 \mathrm{H}, \mathrm{CMe} e_{2}\right) .{ }^{13} \mathrm{C}\left\{{ }^{1} \mathrm{H}\right\} \mathrm{NMR}\left(\mathrm{CDCl}_{3}, 75.46 \mathrm{MHz}, \delta\right): 172.93,134.71$, 117.66, 60.97, 44.81, 39.23, 35.23, 24.69, 16.09. IR $\left(\mathrm{CH}_{2} \mathrm{Cl}_{2}\right.$ soln, $\left.v_{\mathrm{C}=\mathrm{N}}, \mathrm{cm}^{-1}\right): 1664.6$. 
General procedure for the synthesis of catalysts $17 \mathbf{a}-\mathbf{c}$

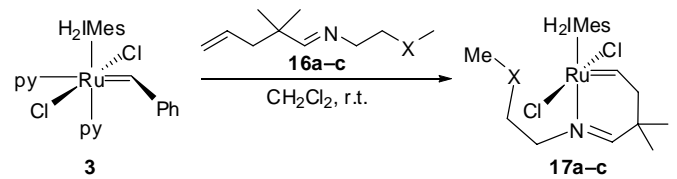

In the glove box, a Schlenk flask was charged with 3 and $\mathrm{CH}_{2} \mathrm{Cl}_{2}$. The corresponding imine was then added via syringe and the reaction stirred at r.t. for $30 \mathrm{~min}$. The volatiles were removed under vacuum, the residue redissolved in $\mathrm{C}_{6} \mathrm{H}_{6}(2 \mathrm{~mL})$, and precipitated with pentane $(20 \mathrm{~mL})$, cooling to $-5{ }^{\circ} \mathrm{C}$. The solid was collected, washed with pentane $(3 \times 5 \mathrm{~mL})$ and dried under vacuum to give the imine-substituted ruthenium compounds in good yields. Any modifications are described below for each reaction.

$\mathrm{X}=\mathrm{CH}_{2}$ (17a)

Ru complex 3 (140 mg, $0.192 \mathrm{mmol}$ ), imine 16a (48.0 mg, $0.219 \mathrm{mmol})$ and $\mathrm{CH}_{2} \mathrm{Cl}_{2}(5 \mathrm{~mL})$ gave 17a (85.3 mg, $0.135 \mathrm{mmol})$ as a light green solid. Yield: $70 \%$. ${ }^{1} \mathrm{H} \mathrm{NMR}\left(\mathrm{CD}_{2} \mathrm{Cl}_{2}, 299.87\right.$ $\mathrm{MHz}, \delta$ ): 18.71 (t, $J=5.4 \mathrm{~Hz}, 1 \mathrm{H}, \mathrm{Ru}=\mathrm{CHCH}_{2}$ ), 7.38 (t, $\left.J=1.2 \mathrm{~Hz}, 1 \mathrm{H}, \mathrm{CH}=\mathrm{N}\right), 7.00$ (br s, 4 H, Mes), 4.03 (s, $4 \mathrm{H}, \mathrm{NCH}_{2} \mathrm{CH}_{2} \mathrm{~N}$ ), 3.03 (td, $J=7.8,1.2 \mathrm{~Hz}, 2 \mathrm{H}, \mathrm{CH}=\mathrm{NCH}_{2} \mathrm{CH}_{2}$ ), $2.84(\mathrm{~d}, J=$ $5.4 \mathrm{~Hz}, 2 \mathrm{H}, \mathrm{Ru}=\mathrm{CHCH}_{2} \mathrm{CMe}_{2}$ ), 2.42 (br s, $12 \mathrm{H}, \mathrm{Mes}-\mathrm{CH}_{3}$ ), 2.34 (s, $6 \mathrm{H}, \mathrm{Mes}-\mathrm{CH}_{3}$ ), 1.19 (m, 2 $\mathrm{H}, \mathrm{NCH}_{2} \mathrm{CH}_{2} \mathrm{CH}_{2}$ ), 1.04 (sext., $J=7.8 \mathrm{~Hz}, \mathrm{CH}_{2} \mathrm{CH}_{2} \mathrm{Me}$ ), 0.93 (s, $6 \mathrm{H}, \mathrm{CMe}_{2}$ ), 0.77 (t, $J=7.5 \mathrm{~Hz}$, $3 \mathrm{H}, \mathrm{Bu}-\mathrm{Me}) .{ }^{13} \mathrm{C}\left\{{ }^{1} \mathrm{H}\right\}$ NMR $\left(\mathrm{CD}_{2} \mathrm{Cl}_{2}, 125.71 \mathrm{MHz}, \delta\right): 344.20\left(\mathrm{Ru}=\mathrm{CHCH} \mathrm{H}_{2}\right), 219.23$ $\left(\mathrm{Ru}-\mathrm{C}(\mathrm{N})_{2}\right), 174.25(\mathrm{Ru}-\mathrm{N}=C), 138.84,129.74$, 64.54, 61.70, 51.72 (br), 42.00, 31.14, 27.04, 21.40, 20.89, $19.58(\mathrm{br})$, 13.61. IR $\left(\mathrm{CH}_{2} \mathrm{Cl}_{2}\right.$ soln, $\left.v_{\mathrm{C}=\mathrm{N}}, \mathrm{cm}^{-1}\right): 1634.9$. HRMS-FAB (m/z): [M] ${ }^{+}$ calcd for $\mathrm{C}_{31} \mathrm{H}_{45} \mathrm{Cl}_{2} \mathrm{~N}_{3} \mathrm{Ru}$, 631.2035; found, 631.2042.

\section{$\mathrm{X}=\mathrm{O}(\mathbf{1 7 b})$}

Ru complex 3 (160 mg, $0.220 \mathrm{mmol}$ ), imine 16b (46.8 mg, $0.276 \mathrm{mmol})$ and $\mathrm{CH}_{2} \mathrm{Cl}_{2}(5 \mathrm{~mL})$ gave 17b (115.8 mg, $0.182 \mathrm{mmol}$ ) as a light green solid. Yield: $83 \%$. ${ }^{1} \mathrm{H} \mathrm{NMR}\left(\mathrm{CD}_{2} \mathrm{Cl}_{2}, 299.87\right.$ $\mathrm{MHz}, \delta$ ): 18.64 (t, $J=5.7 \mathrm{~Hz}, 1 \mathrm{H}, \mathrm{Ru}=\mathrm{CHCH}_{2}$ ), 7.47 (t, $\left.J=1.5 \mathrm{~Hz}, 1 \mathrm{H}, \mathrm{CH}=\mathrm{N}\right), 7.00$ (s, $4 \mathrm{H}$, Mes), 3.93 (s, $4 \mathrm{H}, \mathrm{NCH}_{2} \mathrm{CH}_{2} \mathrm{~N}$ ), 3.53 (t, $J=5.4 \mathrm{~Hz}, 2 \mathrm{H}, \mathrm{NCH}_{2} \mathrm{CH}_{2} \mathrm{OMe}$ ), 3.14 (td, $J=5.8,1.5$ $\mathrm{Hz}, 2 \mathrm{H}, \mathrm{CH}=\mathrm{NCH}_{2} \mathrm{CH}_{2}$ ), 2.92 (d, $J=5.4 \mathrm{~Hz}, 2 \mathrm{H}, \mathrm{Ru}=\mathrm{CHCH}_{2} \mathrm{CMe}_{2}$ ), 2.83 (s, $3 \mathrm{H}, \mathrm{OMe}$ ), 2.44 (s, $\left.12 \mathrm{H}, \mathrm{Mes}-\mathrm{CH}_{3}\right), 2.35$ (s, $6 \mathrm{H}$, Mes-CH $\mathrm{CH}_{3}, 0.95$ (s, $\left.6 \mathrm{H}, \mathrm{CMe}\right)_{2}{ }^{13} \mathrm{C}\left\{{ }^{1} \mathrm{H}\right\} \mathrm{NMR}\left(\mathrm{CD}_{2} \mathrm{Cl}_{2}, 75.42\right.$ $\mathrm{MHz}, \delta): 341.29\left(\mathrm{Ru}=\mathrm{CHCH}_{2}\right), 218.88\left(\mathrm{Ru}-\mathrm{C}(\mathrm{N})_{2}\right), 176.24(\mathrm{Ru}-\mathrm{N}=\mathrm{C}), 139.03,138.64,137.40$, 129.73, 70.46, 63.31, 59.33, 58.61, 51.95, 40.89, 26.96, 21.35, 19.49. IR $\left(\mathrm{CH}_{2} \mathrm{Cl}_{2}\right.$ soln, $v_{\mathrm{C}=\mathrm{N}}$, $\mathrm{cm}^{-1}$ ): 1645.8. HRMS-FAB (m/z): [M] ${ }^{+}$calcd for $\mathrm{C}_{30} \mathrm{H}_{43} \mathrm{Cl}_{2} \mathrm{~N}_{3} \mathrm{RuO}$, 633.1827; found, 633.1845.

$\mathrm{X}=\mathrm{S}(\mathbf{1 7} \mathrm{c})$

Ru complex 3 (149 mg, $0.205 \mathrm{mmol})$, imine 16c (47.0 mg, $0.253 \mathrm{mmol})$ and $\mathrm{CH}_{2} \mathrm{Cl}_{2}(5 \mathrm{~mL})$ gave 17c (110.2 mg, $0.169 \mathrm{mmol})$ as a light green solid. Yield: $83 \%$. ${ }^{1} \mathrm{H} \mathrm{NMR}\left(\mathrm{CD}_{2} \mathrm{Cl}_{2}, 299.82\right.$ $\mathrm{MHz}, \delta$ ): 18.45 (t, $J=7.5 \mathrm{~Hz}, 1 \mathrm{H}, \mathrm{Ru}=\mathrm{CHCH}_{2}$ ), 7.47 (s, $1 \mathrm{H}, \mathrm{CH}=\mathrm{N}$ ), 7.01 (s, $\left.4 \mathrm{H}, \mathrm{Mes}\right), 3.79$ $\left(\mathrm{s}, 4 \mathrm{H}, \mathrm{NCH}_{2} \mathrm{CH}_{2} \mathrm{~N}\right), 3.13\left(\mathrm{~m}, 4 \mathrm{H}, \mathrm{NCH}_{2} \mathrm{CH}_{2}\right.$ and $\left.\mathrm{Ru}=\mathrm{CHCH}_{2} \mathrm{CMe}_{2}\right), 2.5-2.4(\mathrm{~m}, 2 \mathrm{H}$, $\mathrm{NCH}_{2} \mathrm{CH}_{2} \mathrm{SMe}$ ), 2.42 (s, $12 \mathrm{H}$, Mes- $\mathrm{CH}_{3}$ ), 2.37 (s, $6 \mathrm{H}, \mathrm{Mes}-\mathrm{CH}_{3}$ ), 1.43 (s, $3 \mathrm{H}, \mathrm{CH}_{2} \mathrm{SMe}$ ), 0.95 
(s, $\left.6 \mathrm{H}, \mathrm{CMe}_{2}\right) \cdot{ }^{13} \mathrm{C}\left\{{ }^{1} \mathrm{H}\right\}$ NMR $\left(\mathrm{CD}_{2} \mathrm{Cl}_{2}, 75.42 \mathrm{MHz}, \delta\right): 342.16\left(\mathrm{Ru}=\mathrm{CHCH}_{2}\right), 219.28$ $\left(\mathrm{Ru}-\mathrm{C}(\mathrm{N})_{2}\right), 175.04(\mathrm{Ru}-\mathrm{N}=C), 139.14,138.90,138.44,129.96,61.52,60.61,51.89,38.58$, 35.32, 27.04, 21.40, 19.40, 14.44. IR $\left(\mathrm{CH}_{2} \mathrm{Cl}_{2}\right.$ soln, $\left.v_{\mathrm{C}=\mathrm{N}}, \mathrm{cm}^{-1}\right)$ : 1641.3. HRMS-FAB (m/z): $[\mathrm{M}]^{+}$calcd for $\mathrm{C}_{30} \mathrm{H}_{43} \mathrm{Cl}_{2} \mathrm{~N}_{3} \mathrm{RuS}$, 649.1599; found, 649.1626.

\section{Catalysis Procedures}

RCM of Diethyldiallyl Malonate (10) at elevated temperature

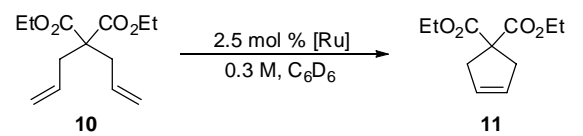

A stock solution was prepared to deliver the catalyst solution. Inside a glovebox, a volumetric flask was charged with catalyst $(0.026 \mathrm{mmol})$ and $\mathrm{C}_{6} \mathrm{D}_{6}$ added to prepare $2.0 \mathrm{~mL}$ of stock solution $(0.013 \mathrm{M})$. An NMR tube with a screwcap septum top was charged with catalyst stock solution $(0.013 \mathrm{M}, 0.40 \mathrm{~mL}, 5.2 \mu \mathrm{mol}, 2.5 \mathrm{~mol} \%)$ and $\mathrm{C}_{6} \mathrm{D}_{6}(0.25 \mathrm{~mL})$. The sample was equilibrated at the desired temperature in the NMR probe before 10 (50 $\mu \mathrm{L}, 50 \mathrm{mg}, 0.21 \mathrm{mmol}$, $0.3 \mathrm{M}$ ) was added via syringe. Data points were collected over an appropriate period of time using the Varian array function. The conversion to $\mathbf{1 1}$ was determined by comparing the ratio of the integrals of the methylene protons in the starting material, $\delta 2.84$ (dt), with those in the product, $\delta 3.14$ (s).

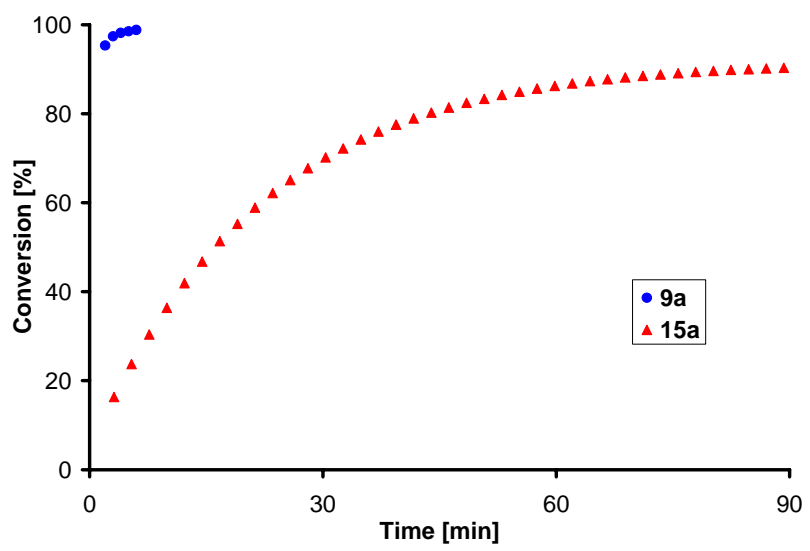

Figure S1. Conversion plot for RCM of 10 with 9a, $15 a\left(2.5 \mathrm{~mol} \mathrm{\% ,} 30{ }^{\circ} \mathrm{C}, 0.3 \mathrm{M} \mathrm{C}_{6} \mathrm{D}_{6}\right)$ 
ROMP of Dicyclopentadiene (DCPD).

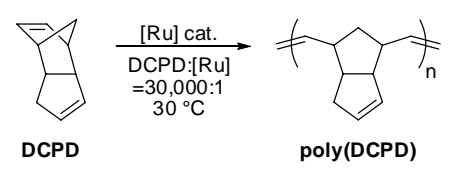

DCPD containing 3.5\% of tricyclopentadiene (100 g) was polymerized by addition of catalyst $(0.025 \mathrm{mmol}$, monomer/catalyst $=30,000)$ in a minimal amount of $\mathrm{CH}_{2} \mathrm{Cl}_{2}$ at $30{ }^{\circ} \mathrm{C}$. The reaction was monitored by measuring the polymerization exotherm with a temperature probe.

RCM of Diethyldiallyl Malonate (10) to test latency

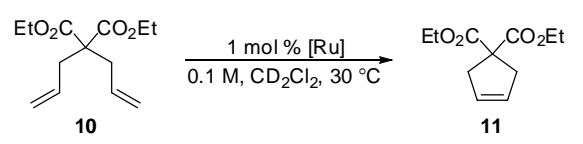

A stock solution was prepared to deliver the catalyst solution. Inside a glovebox, a volumetric flask was charged with catalyst $(0.016 \mathrm{mmol})$ and $\mathrm{CD}_{2} \mathrm{Cl}_{2}$ added to prepare $1.0 \mathrm{~mL}$ of stock solution $(0.016 \mathrm{M})$. An NMR tube with a screwcap septum top was charged with catalyst stock solution $(0.016 \mathrm{M}, 50 \mu \mathrm{L}, 0.80 \mu \mathrm{mol}, 1.0 \mathrm{~mol} \%)$ and $\mathrm{CD}_{2} \mathrm{Cl}_{2}(0.75 \mathrm{~mL})$. The sample was equilibrated at $30^{\circ} \mathrm{C}$ in the NMR probe before $10(19.3 \mu \mathrm{L}, 19.2 \mathrm{mg}, 0.080 \mathrm{mmol}, 0.1 \mathrm{M})$ was added via syringe. Data points were collected over an appropriate period of time using the Varian array function. The conversion to $\mathbf{1 1}$ was determined by comparing the ratio of the integrals of the methylene protons in the starting material, $\delta 2.61(\mathrm{dt})$, with those in the product, $\delta 2.98$ (s).

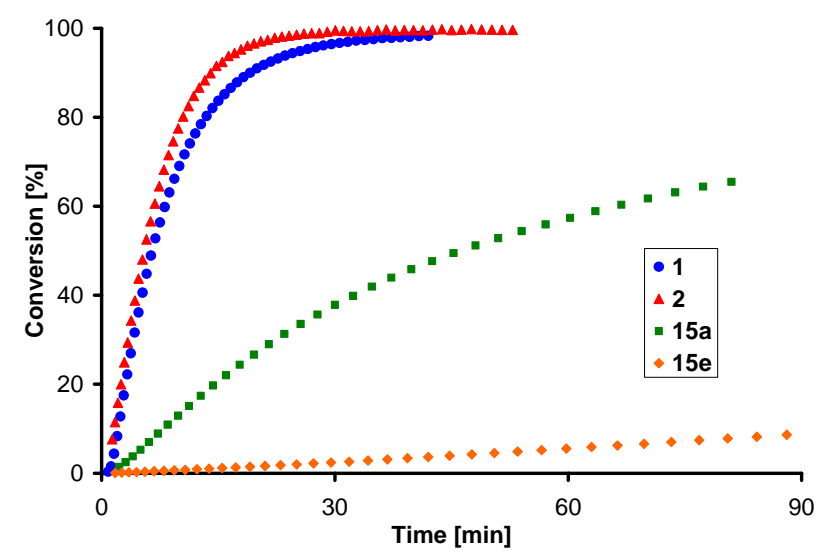

Figure S2. Conversion plot for RCM of 10 with 1, 2, 15a, 15e $\left(1 \mathrm{~mol} \%, 30^{\circ} \mathrm{C}, 0.1 \mathrm{M} \mathrm{CD}_{2} \mathrm{Cl}_{2}\right)$ 
Test of sulfur inhibition

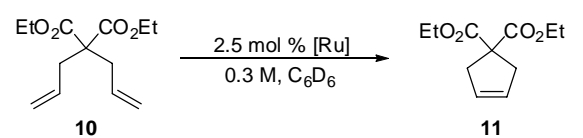

A stock solution was prepared to deliver the catalyst solution. Inside a glovebox, a volumetric flask was charged with catalyst $17 \mathbf{a}(0.026 \mathrm{mmol})$ and $\mathrm{C}_{6} \mathrm{D}_{6}$ added to prepare $2.0 \mathrm{~mL}$ of catalyst stock solution $(0.013 \mathrm{M})$. A stock solution of $\mathrm{SMe}_{2}$ was prepared by dissolving $\mathrm{SMe}_{2}(30.3 \mu \mathrm{L}$, $25.6 \mathrm{mg}, 0.41 \mathrm{mmol})$ and $\mathrm{C}_{6} \mathrm{D}_{6}$ added to prepare $2.0 \mathrm{~mL}$ of solution $(0.21 \mathrm{M})$. An NMR tube with a screwcap septum top was charged with catalyst stock solution $(0.013 \mathrm{M}, 0.40 \mathrm{~mL}, 5.2$ $\mu \mathrm{mol}, 2.5 \mathrm{~mol} \%), \mathrm{SMe}_{2}$ stock solution $(25 \mu \mathrm{L}, 5.2 \mu \mathrm{mol})$ and $\mathrm{C}_{6} \mathrm{D}_{6}(0.25 \mathrm{~mL})$. The sample was equilibrated at $60{ }^{\circ} \mathrm{C}$ in the NMR probe before $10(50 \mu \mathrm{L}, 50 \mathrm{mg}, 0.21 \mathrm{mmol}, 0.3 \mathrm{M})$ was added via syringe. Data points were collected over an appropriate period of time using the Varian array function. The conversion to $\mathbf{1 1}$ was determined by comparing the ratio of the integrals of the methylene protons in the starting material, $\delta 2.84(\mathrm{dt})$, with those in the product, $\delta 3.14$ (s). Corresponding NMR samples were prepared with 17a and 17c in the absence of $\mathrm{SMe}_{2}$.

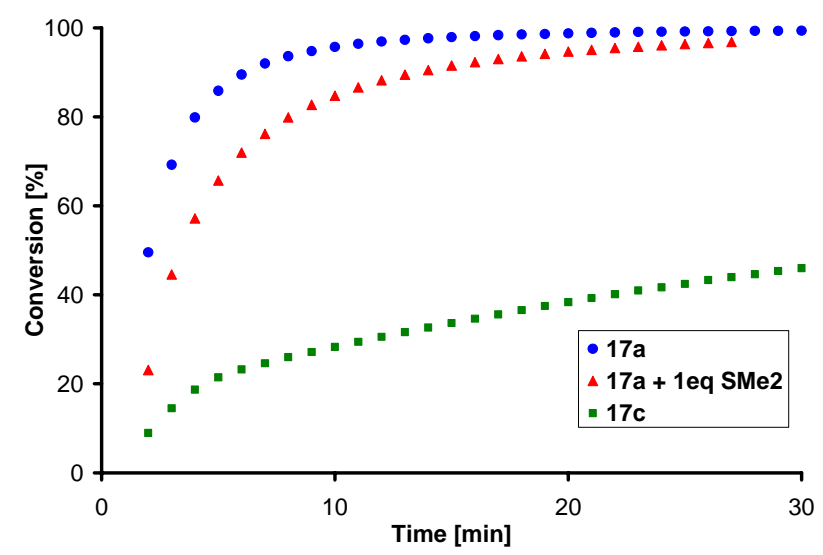

Figure S3. Conversion plot for RCM of 10 with 17a, 17a $+\mathrm{SMe}_{2}, 17 \mathrm{c}\left(2.5 \mathrm{~mol} \%, 65{ }^{\circ} \mathrm{C}, 0.3 \mathrm{M}\right.$ $\left.\mathrm{C}_{6} \mathrm{D}_{6}\right)$

\section{X-ray Crystallography}

Crystallographic data have been deposited at the CCDC, 12 Union Road, Cambridge CB2 1EZ, UK and copies can be obtained on request, free of charge, by quoting the publication citation and the deposition number - 9a (CCDC 278062), 15a (CCDC 254980), 15e (CCDC 272587), 17c (CCDC 281471). 
Table S1. Crystal data and structure refinement for 9a (CCDC 278062).

Empirical formula

Formula weight

Crystallization Solvent

Crystal Habit

Crystal size

Crystal color

Type of diffractometer

Wavelength

Data Collection Temperature

$\theta$ range for 14476 reflections used

in lattice determination

Unit cell dimensions

Volume

Z

Crystal system

Space group

Density (calculated)

$\mathrm{F}(000)$

Data collection program

$\theta$ range for data collection

Completeness to $\theta=27.55^{\circ}$

Index ranges

Data collection scan type

Data reduction program

Reflections collected

Independent reflections

Absorption coefficient

Absorption correction

Max. and min. transmission
$\mathrm{C}_{34} \mathrm{H}_{43} \mathrm{~N}_{3} \mathrm{Cl}_{2} \mathrm{Ru}$

665.68

THF/pentane

Olive brown

$0.28 \times 0.17 \times 0.15 \mathrm{~mm}^{3}$

Block

\section{Data Collection}

Bruker SMART 1000

$0.71073 \AA \mathrm{MoK} \alpha$

100(2) K

2.33 to $32.02^{\circ}$

$\mathrm{a}=13.7555(7) \AA$

$\mathrm{b}=11.4276(6) \AA$

$c=20.4538(11) \AA$

$\beta=98.6480(10)^{\circ}$

3178.6(3) $\AA^{3}$

4

Monoclinic

$\mathrm{P} 2{ }_{1} / \mathrm{n}$

$1.391 \mathrm{Mg} / \mathrm{m}^{3}$

1384

Bruker SMART v5.630

1.67 to $27.55^{\circ}$

$99.6 \%$

$-17 \leq \mathrm{h} \leq 17,-14 \leq \mathrm{k} \leq 14,-26 \leq \mathrm{l} \leq 26$

$\omega$ scans at $5 \phi$ settings

Bruker SAINT v6.45A

42974

$7309\left[\mathrm{R}_{\mathrm{int}}=0.0840\right]$

$0.689 \mathrm{~mm}^{-1}$

None

0.9037 and 0.8305 


\section{Table S1 (cont.)}

\section{Structure solution and Refinement}

Structure solution program

Primary solution method

Secondary solution method

Hydrogen placement

Structure refinement program

Refinement method

Data / restraints / parameters

Treatment of hydrogen atoms

Goodness-of-fit on $\mathrm{F}^{2}$

Final R indices [I $>2 \sigma(\mathrm{I}), 5586$ reflections]

$\mathrm{R}$ indices (all data)

Type of weighting scheme used

Weighting scheme used

Max shift/error

Average shift/error

Largest diff. peak and hole
Bruker XS v6.12

Direct methods

Difference Fourier map

Geometric positions

Bruker XL v6.12

Full matrix least-squares on $\mathrm{F}^{2}$

7309 / 0 / 369

Riding

2.222

$\mathrm{R} 1=0.0593, w \mathrm{R} 2=0.1034$

$\mathrm{R} 1=0.0831, w \mathrm{R} 2=0.1064$

Sigma

$w=1 / \sigma^{2}\left(\mathrm{Fo}^{2}\right)$

0.001

0.000

2.767 and -1.273 e. $\AA^{-3}$

\section{Special Refinement Details}

$\mathrm{Ru}$.

All peaks in the final electron difference Fourier map representing more than one electron lie within $1 \AA$ of

Refinement of $\mathrm{F}^{2}$ against ALL reflections. The weighted R-factor ( $\left.w \mathrm{R}\right)$ and goodness of fit (S) are based on $\mathrm{F}^{2}$, conventional R-factors $(\mathrm{R})$ are based on $\mathrm{F}$, with $\mathrm{F}$ set to zero for negative $\mathrm{F}^{2}$. The threshold expression of $\mathrm{F}^{2}>$ $2 \sigma\left(\mathrm{F}^{2}\right)$ is used only for calculating R-factors(gt) etc. and is not relevant to the choice of reflections for refinement. R-factors based on $\mathrm{F}^{2}$ are statistically about twice as large as those based on $\mathrm{F}$, and R-factors based on ALL data will be even larger.

All esds (except the esd in the dihedral angle between two l.s. planes) are estimated using the full covariance matrix. The cell esds are taken into account individually in the estimation of esds in distances, angles and torsion angles; correlations between esds in cell parameters are only used when they are defined by crystal symmetry. An approximate (isotropic) treatment of cell esds is used for estimating esds involving l.s. planes. 


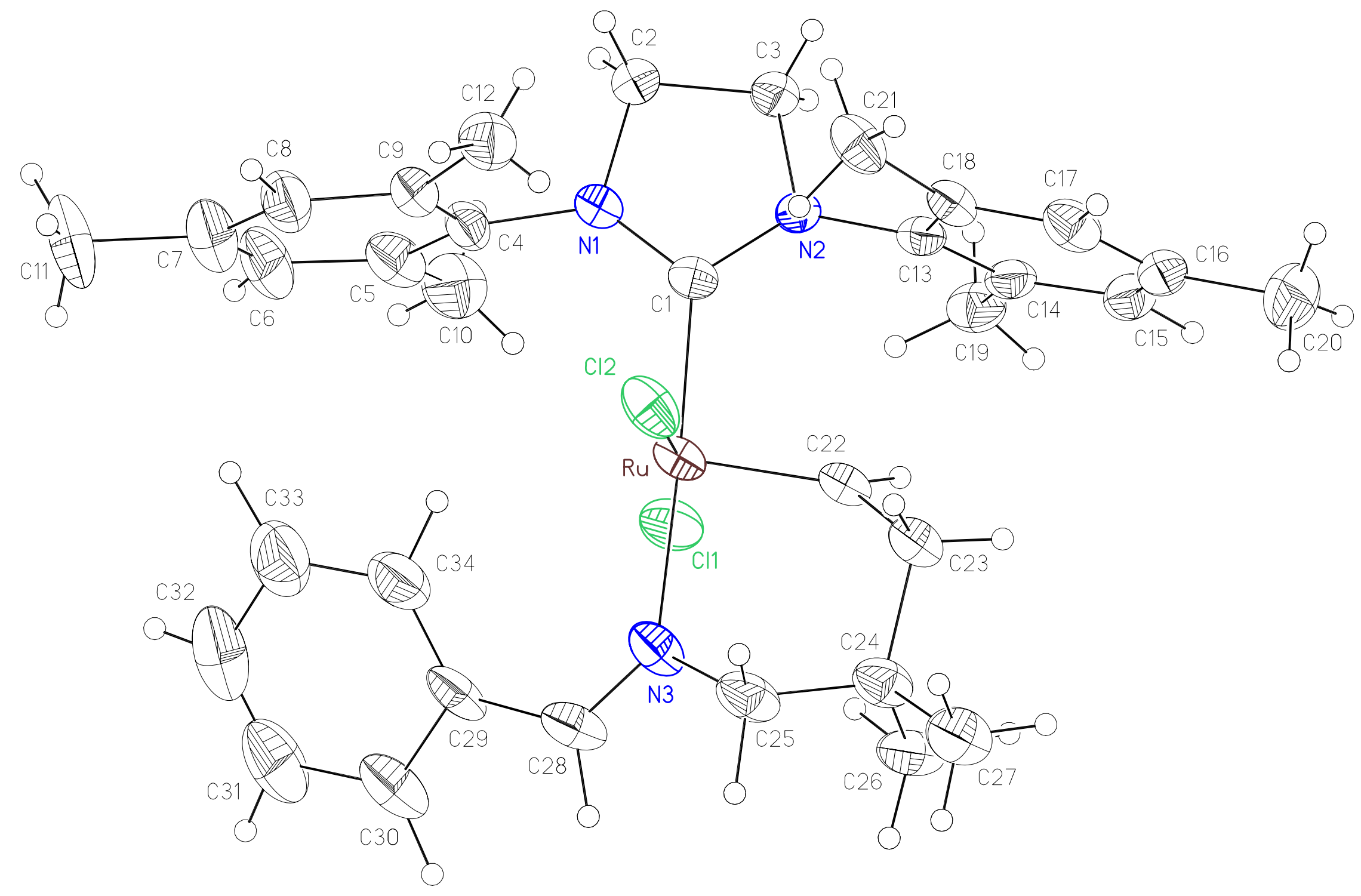

Figure S4. Labeled view of 9a 
Table S2. Crystal data and structure refinement for 15a (CCDC 254980).

Empirical formula

Formula weight

Crystallization Solvent

Crystal Habit

Crystal size

Crystal color

Type of diffractometer

Wavelength

Data Collection Temperature

$\theta$ range for 19323 reflections used

in lattice determination

Unit cell dimensions

Volume

Z

Crystal system

Space group

Density (calculated)

$\mathrm{F}(000)$

$\theta$ range for data collection

Completeness to $\theta=47.34^{\circ}$

Index ranges

Data collection scan type

Reflections collected

Independent reflections

Absorption coefficient

Absorption correction

Max. and min. transmission
$\mathrm{C}_{33} \mathrm{H}_{41} \mathrm{~N}_{3} \mathrm{Cl}_{2} \mathrm{Ru}$

651.66

THF/pentane

Block

$0.33 \times 0.31 \times 0.27 \mathrm{~mm}^{3}$

Dichroic Green/brown

\section{Data Collection}

Bruker SMART 1000

$0.71073 \AA$ MoK $\alpha$

100(2) K

2.32 to $45.20^{\circ}$

$\mathrm{a}=13.5715(4) \AA$

$\mathrm{b}=11.6530(4) \AA$

$\mathrm{c}=20.2953(6) \AA$

$\beta=100.8070(10)^{\circ}$

3152.75(17) $\AA^{3}$

4

Monoclinic

$\mathrm{P} 2{ }_{1} / \mathrm{n}$

$1.373 \mathrm{Mg} / \mathrm{m}^{3}$

1352

1.67 to $47.34^{\circ}$

$80.2 \%$

$-28 \leq \mathrm{h} \leq 24,-24 \leq \mathrm{k} \leq 24,-41 \leq \mathrm{l} \leq 41$

$\omega$ scans at $3 \phi$ settings for $2 \theta=-28^{\circ}$ and 2 for $2 \theta=-70^{\circ}$

62469

$23433\left[\mathrm{R}_{\mathrm{int}}=0.0583\right]$

$0.693 \mathrm{~mm}^{-1}$

None

0.8350 and 0.8036 


\section{Table S2 (cont.)}

\section{Structure solution and Refinement}

Structure solution program

Primary solution method

Secondary solution method

Hydrogen placement

Structure refinement program

Refinement method

Data / restraints / parameters

Treatment of hydrogen atoms

Goodness-of-fit on $\mathrm{F}^{2}$

Final R indices [I $>2 \sigma(\mathrm{I}), 13403$ reflections]

$\mathrm{R}$ indices (all data)

Type of weighting scheme used

Weighting scheme used

Max shift/error

Average shift/error

Largest diff. peak and hole
SHELXS-97 (Sheldrick, 1990)

Direct methods

Difference Fourier map

Geometric positions

SHELXL-97 (Sheldrick, 1997)

Full matrix least-squares on $\mathrm{F}^{2}$

23433 / 0 / 390

Riding

1.358

$\mathrm{R} 1=0.0514, w \mathrm{R} 2=0.0848$

$\mathrm{R} 1=0.0974, w \mathrm{R} 2=0.0898$

Sigma

$w=1 / \sigma^{2}\left(\mathrm{Fo}^{2}\right)$

0.004

0.000

2.906 and -2.223 e. $\AA^{-3}$

\section{Special Refinement Details}

The molecule exhibits disorder in the link between C22 and N3 where both possible pucker orientations are observed. The methylene carbon of the linkage (C23) adopts two different positions which correlate to alternate positions for the two methyl groups (C25 and C26) bonded to C24. The ratio between both conformations is 0.68:0.32 (see Table 2). The third figure illustrates both, the minor component in blue with dashed bonds.

All hydrogen atoms were restrained to ride the respective carbon but methyl groups were allowed to rotate about the C-C bond to provide maximum fit to the observed electron density. A cluster of difference electron density peaks (greater than $1 \mathrm{e}-/ \AA^{3}$ ) appear within $1 \AA$ of $\mathrm{Ru}$ in the final map.

Refinement of $\mathrm{F}^{2}$ against ALL reflections. The weighted R-factor $(w \mathrm{R})$ and goodness of fit $(\mathrm{S})$ are based on $\mathrm{F}^{2}$, conventional R-factors $(\mathrm{R})$ are based on $\mathrm{F}$, with $\mathrm{F}$ set to zero for negative $\mathrm{F}^{2}$. The threshold expression of $\mathrm{F}^{2}>$ $2 \sigma\left(\mathrm{F}^{2}\right)$ is used only for calculating R-factors(gt) etc. and is not relevant to the choice of reflections for refinement. R-factors based on $\mathrm{F}^{2}$ are statistically about twice as large as those based on F, and R-factors based on ALL data will be even larger.

All esds (except the esd in the dihedral angle between two l.s. planes) are estimated using the full covariance matrix. The cell esds are taken into account individually in the estimation of esds in distances, angles and torsion angles; correlations between esds in cell parameters are only used when they are defined by crystal symmetry. An approximate (isotropic) treatment of cell esds is used for estimating esds involving l.s. planes. 


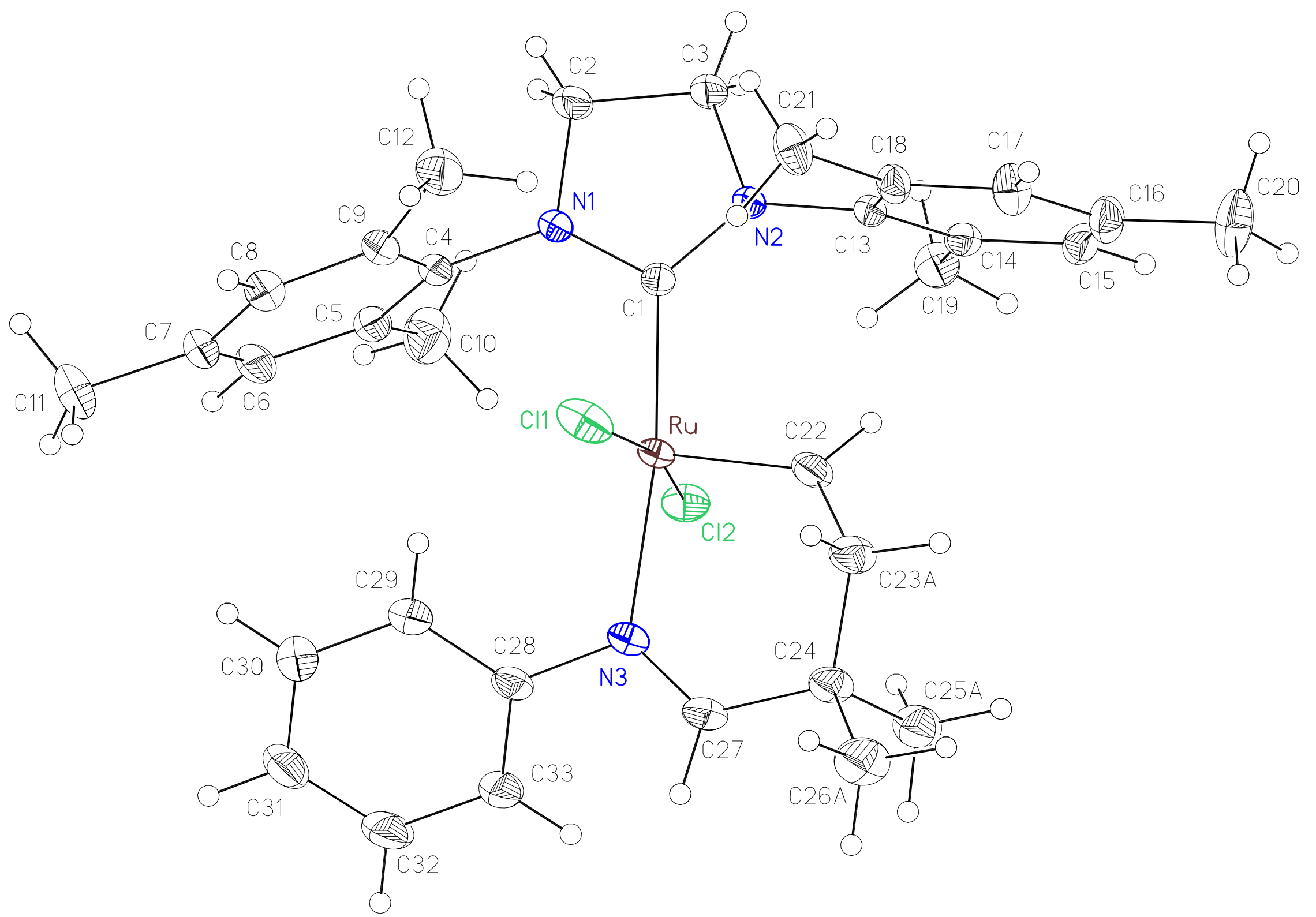

Figure S5. Labeled view of 15a, conformation A 


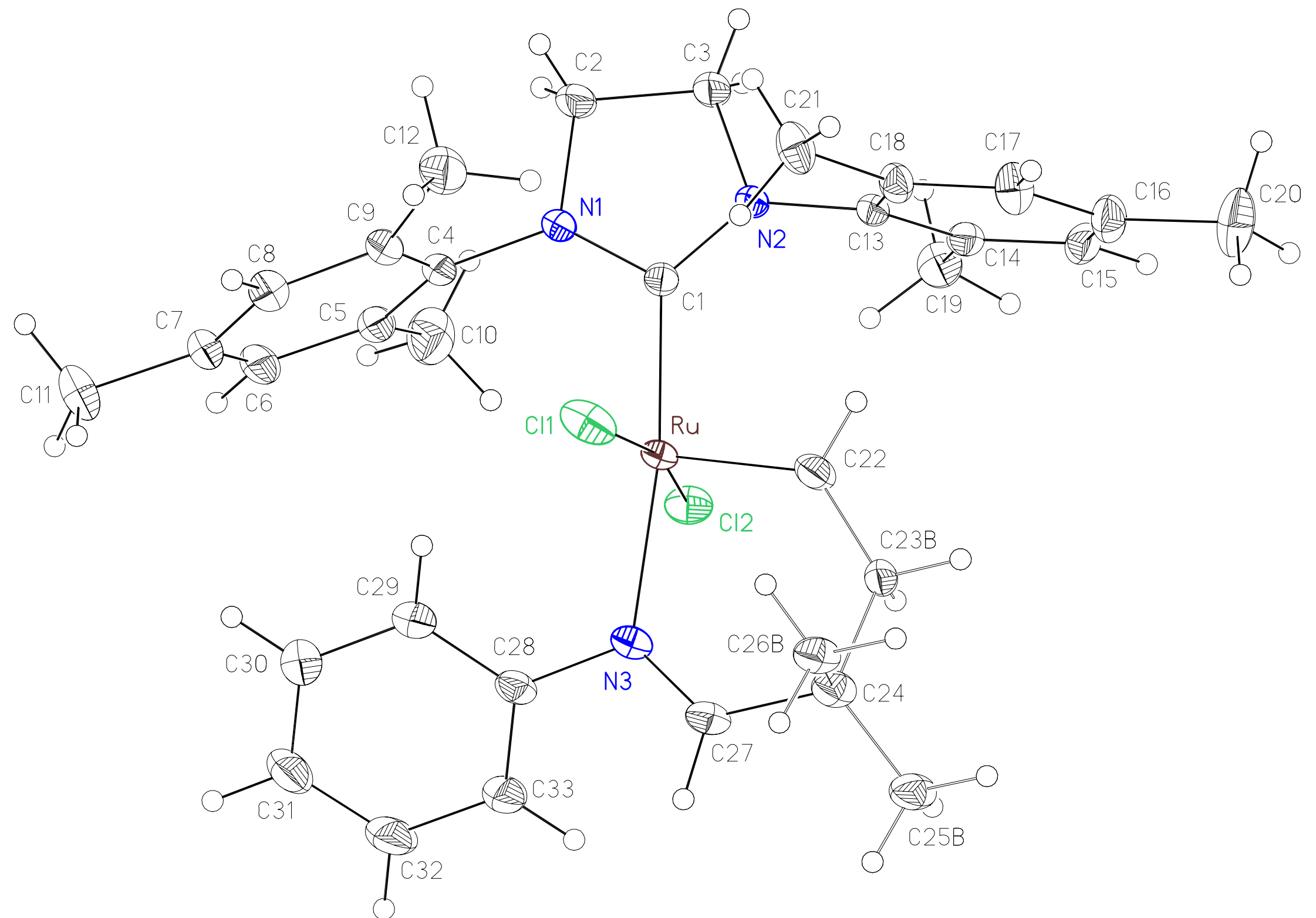

Figure S6. Labeled view of 15a, conformation B 


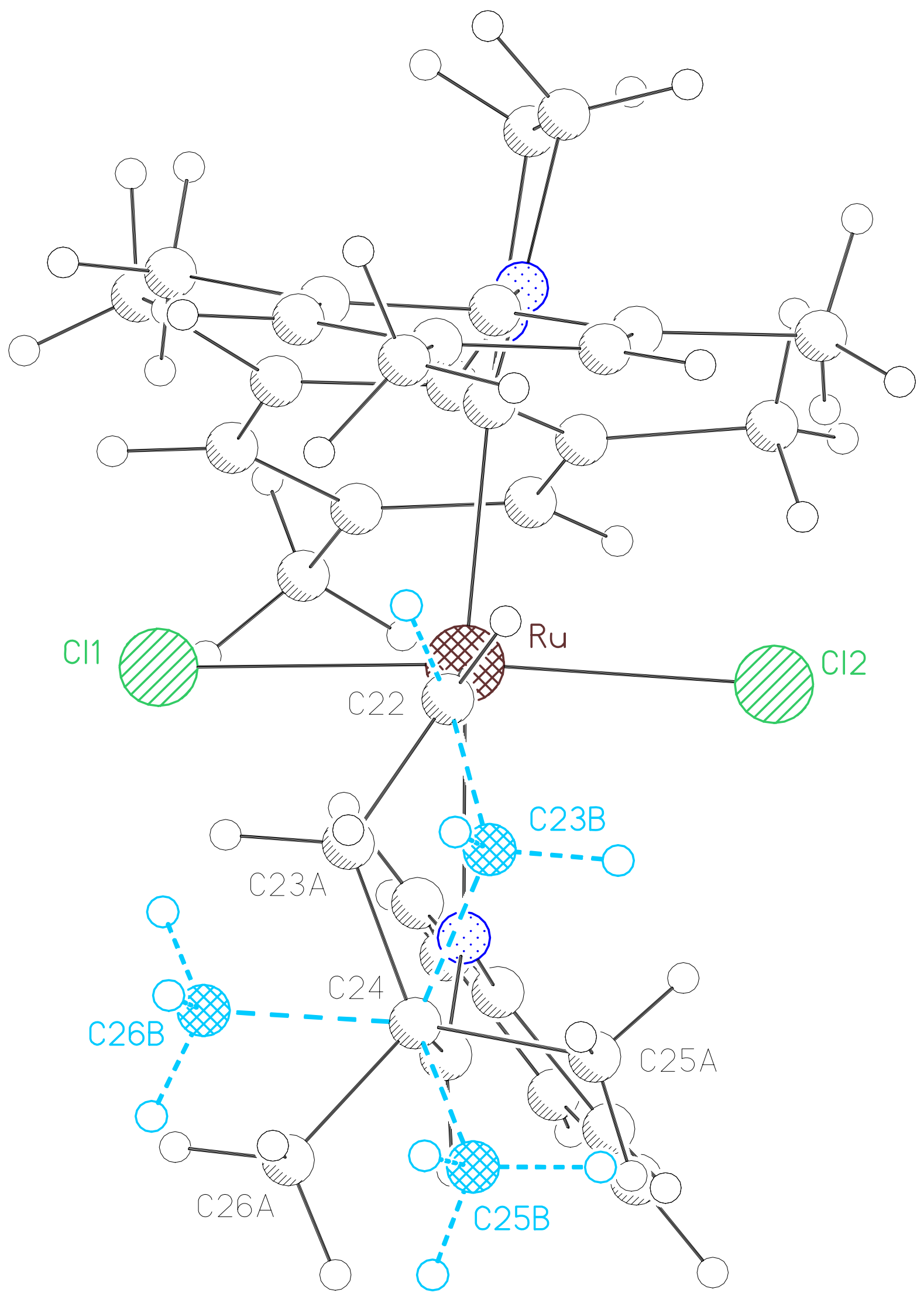

Figure S7. Labeled side view of 15a, conformations A and B overlaid 
Table S3. Crystal data and structure refinement for 15e (CCDC 272587).

Empirical formula

Formula weight

Crystallization Solvent

Crystal Habit

Crystal size

Crystal color

Type of diffractometer

Wavelength

Data Collection Temperature

$\theta$ range for 10966 reflections used

in lattice determination

Unit cell dimensions

Volume

Z

Crystal system

Space group

Density (calculated)

$\mathrm{F}(000)$

Data collection program

$\theta$ range for data collection

Completeness to $\theta=33.52^{\circ}$

Index ranges

Data collection scan type

Data reduction program

Reflections collected

Independent reflections

Absorption coefficient

Absorption correction

Max. and min. transmission
$\mathrm{C}_{28} \mathrm{H}_{39} \mathrm{~N}_{3} \mathrm{Cl}_{2} \mathrm{Ru}$

589.59

THF/hexanes

Fragment

$0.33 \times 0.20 \times 0.10 \mathrm{~mm}^{3}$

Trichroic - green/purple/blue

\section{Data Collection}

Bruker SMART 1000

$0.71073 \AA \mathrm{MoK} \alpha$

100(2) K

2.26 to $32.71^{\circ}$

$\mathrm{a}=14.9244(9) \AA$

$\mathrm{b}=16.4672(10) \AA$

$\mathrm{c}=11.2788(7) \AA$

2771.9(3) $\AA^{3}$

4

Orthorhombic

Pnnm

$1.413 \mathrm{Mg} / \mathrm{m}^{3}$

1224

Bruker SMART v5.630

1.84 to $33.52^{\circ}$

$95.0 \%$

$-20 \leq \mathrm{h} \leq 22,-24 \leq \mathrm{k} \leq 25,-17 \leq \mathrm{l} \leq 17$

$\omega$ scans at $5 \phi$ settings

Bruker SAINT v6.45A

44687

$5402\left[\mathrm{R}_{\mathrm{int}}=0.1233\right]$

$0.779 \mathrm{~mm}^{-1}$

None

0.9261 and 0.7830 


\section{Table S3 (cont.)}

\section{Structure solution and Refinement}

Structure solution program

Primary solution method

Secondary solution method

Hydrogen placement

Structure refinement program

Refinement method

Data / restraints / parameters

Treatment of hydrogen atoms

Goodness-of-fit on $\mathrm{F}^{2}$

Final R indices [I $>2 \sigma(\mathrm{I}), 3250$ reflections]

$\mathrm{R}$ indices (all data)

Type of weighting scheme used

Weighting scheme used

Max shift/error

Average shift/error

Largest diff. peak and hole
SHELXS-97 (Sheldrick, 1990)

Direct methods

Difference Fourier map

Geometric positions

SHELXL-97 (Sheldrick, 1997)

Full matrix least-squares on $\mathrm{F}^{2}$

5402 / 0 / 209

Riding

1.576

$\mathrm{R} 1=0.0575, w \mathrm{R} 2=0.0784$

$\mathrm{R} 1=0.1089, w \mathrm{R} 2=0.0843$

Sigma

$w=1 / \sigma^{2}\left(\mathrm{Fo}^{2}\right)$

0.010

0.001

1.599 and -2.701 e. $\AA^{-3}$

\section{Special Refinement Details}

The molecule sits on a special position, astride the mirror plane at $z=0$. Therefore, atoms $\mathrm{Ru}(1), \mathrm{N}(1)$, $\mathrm{N}(2), \mathrm{N}(3), \mathrm{C}(1), \mathrm{C}(4), \mathrm{C}(7), \mathrm{C}(9), \mathrm{C}(10), \mathrm{C}(13), \mathrm{C}(15), \mathrm{C}(16), \mathrm{C}(21)$ and $\mathrm{C}(22)$ have coordinates with $z=0$; the mirror plane generates equivalent positions for all other atoms. For $\mathrm{Cl}(1), \mathrm{C}(5), \mathrm{C}(6), \mathrm{C}(8), \mathrm{C}(11), \mathrm{C}(12)$ and $\mathrm{C}(14)$ the mirror supplies positions to complete the unique molecule. For C(2), C(3), C(17), C(18), C(19) and C(20) the mirror produces an alternate conformation of the molecule.

Refinement of $\mathrm{F}^{2}$ against ALL reflections. The weighted R-factor $(w \mathrm{R})$ and goodness of fit $(\mathrm{S})$ are based on $\mathrm{F}^{2}$, conventional R-factors $(\mathrm{R})$ are based on $\mathrm{F}$, with $\mathrm{F}$ set to zero for negative $\mathrm{F}^{2}$. The threshold expression of $\mathrm{F}^{2}>$ $2 \sigma\left(\mathrm{F}^{2}\right)$ is used only for calculating R-factors(gt) etc. and is not relevant to the choice of reflections for refinement. R-factors based on $\mathrm{F}^{2}$ are statistically about twice as large as those based on $\mathrm{F}$, and R-factors based on ALL data will be even larger.

All esds (except the esd in the dihedral angle between two l.s. planes) are estimated using the full covariance matrix. The cell esds are taken into account individually in the estimation of esds in distances, angles and torsion angles; correlations between esds in cell parameters are only used when they are defined by crystal symmetry. An approximate (isotropic) treatment of cell esds is used for estimating esds involving l.s. planes. 


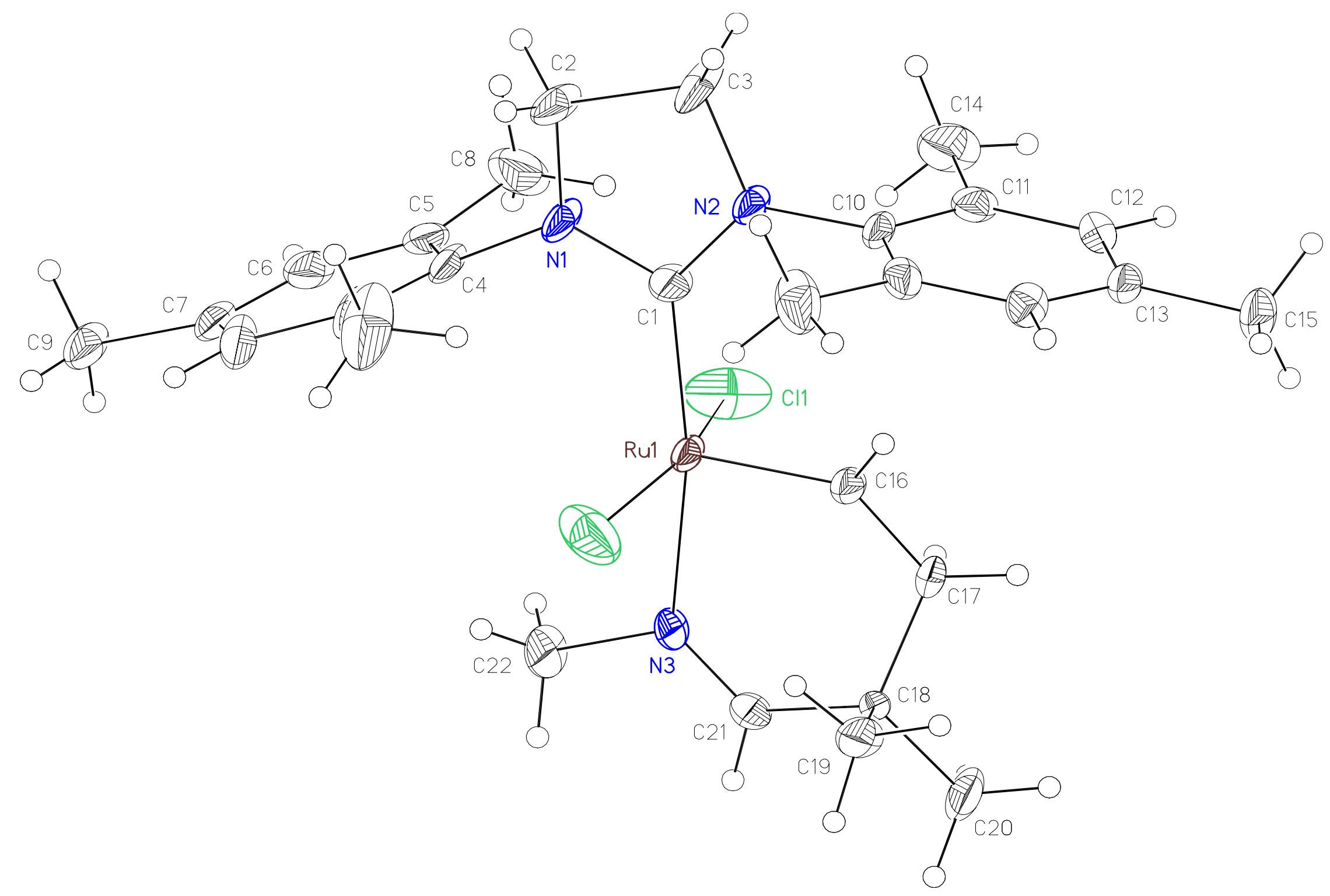

Figure S8. Labeled view of 15e 
Table S4. Crystal data and structure refinement for 17c (CCDC 281471).

Empirical formula

Formula weight

Crystallization Solvent

Crystal Habit

Crystal size

Crystal color

Type of diffractometer

Wavelength

Data Collection Temperature

$\theta$ range for 42363 reflections used

in lattice determination

Unit cell dimensions

Volume

Z

Crystal system

Space group

Density (calculated)

$\mathrm{F}(000)$

$\theta$ range for data collection

Completeness to $\theta=38.31^{\circ}$

Index ranges

Data collection scan type

Reflections collected

Independent reflections

Absorption coefficient

Absorption correction

Max. and min. transmission
$\mathrm{C}_{30} \mathrm{H}_{43} \mathrm{~N}_{3} \mathrm{SCl}_{2} \mathrm{Ru} \cdot 1 / 2\left(\mathrm{C}_{4} \mathrm{H}_{8} \mathrm{O}\right)$

685.76

Pentane/THF

Block

$0.31 \times 0.29 \times 0.29 \mathrm{~mm}^{3}$

Greenblue

\section{Data Collection}

Bruker SMART 1000

$0.71073 \AA \mathrm{MoK} \alpha$

100(2) K

2.22 to $37.63^{\circ}$

$\mathrm{a}=11.1365(3) \AA$

$\mathrm{b}=17.4341(4) \AA$

$\mathrm{c}=34.7895(9) \AA$

$\beta=93.7070(10)^{\circ}$

6740.4(3) $\AA^{3}$

8

Monoclinic

Pc

$1.352 \mathrm{Mg} / \mathrm{m}^{3}$

2864

1.17 to $38.31^{\circ}$

$88.6 \%$

$-18 \leq \mathrm{h} \leq 18,-30 \leq \mathrm{k} \leq 30,-52 \leq \mathrm{l} \leq 52$

$\omega$ scans at $5 \phi$ settings

129948

$58933\left[\mathrm{R}_{\mathrm{int}}=0.0684\right]$

$0.712 \mathrm{~mm}^{-1}$

None

0.8201 and 0.8094 


\section{Table S4 (cont.)}

\section{Structure solution and Refinement}

Structure solution program

Primary solution method

Secondary solution method

Hydrogen placement

Structure refinement program

Refinement method

Data / restraints / parameters

Treatment of hydrogen atoms

Goodness-of-fit on $\mathrm{F}^{2}$

Final R indices [I $>2 \sigma(\mathrm{I}), 41916$ reflections]

$\mathrm{R}$ indices (all data)

Type of weighting scheme used

Weighting scheme used

Max shift/error

Average shift/error

Absolute structure parameter

Largest diff. peak and hole
Bruker XS v6.12

Direct methods

Difference Fourier map

Geometric positions

Bruker XS v6.12

Full matrix least-squares on $\mathrm{F}^{2}$

58933 / 372 / 1573

Riding

1.302

$\mathrm{R} 1=0.0580, w \mathrm{R} 2=0.0946$

$\mathrm{R} 1=0.0900, w \mathrm{R} 2=0.1005$

Sigma

$w=1 / \sigma^{2}\left(\mathrm{Fo}^{2}\right)$

0.002

0.000

0.044(18)

2.556 and -2.245 e. $\AA^{-3}$

\section{Special Refinement Details}

There are four Ru molecules and two molecules of THF in the asymmetric unit. An approximate center of symmetry exists at $x=0.45, y=0.25$ and $z=0.38$ and if actually present would result in space group $\mathrm{P} 2{ }_{1} / \mathrm{c}$. The four $\mathrm{Ru}$ molecules are identical with the exception that one site (molecule $\mathrm{C}$ ) is disordered about an approximate mirror plane through $\mathrm{C}(1 \mathrm{C}), \mathrm{Ru}(3), \mathrm{Cl}(1 \mathrm{C})$ and $\mathrm{Cl}(2 \mathrm{C})$ to give molecule $\mathrm{G}$ (see Figures 3-7.). The atomic positions of $\mathrm{S}(1 \mathrm{C}), \mathrm{N}(3 \mathrm{C})$ and $\mathrm{C}(22 \mathrm{C})-\mathrm{C}(30 \mathrm{C})$ were modeled to give an approximate mirror image composed of atomic positions for $S(1 G), N(3 G)$ and $C(22 G)-C(30 G)$ in a ratio of 61:39 (see Table 1.)

Restraints were required to maintain satisfactory geometry in the disordered ligand of molecule G and were applied as follows; 1 ) all similar bonds (1-2 distances) in each molecule were assigned a target value, 2) all similar angles (1-3 distances) were assigned a target value and 3) target values were refined as free variables during least squares. As a result the model target values are an average of the distances in all four molecules. In this way the model is allowed to define its own geometry without introducing undo bias. The assignment and final target values follow;

\begin{tabular}{llr} 
Bond type & \multicolumn{1}{c}{ Atoms } & Distance \\
\hline $\mathrm{Ru}=\mathrm{Csp}^{2}$ & $\mathrm{Ru} *-\mathrm{C}\left(22^{*}\right)$ & $1.884(5)$ \\
$\mathrm{Csp}^{2}-\mathrm{Csp}^{3}, \mathrm{~N}-\mathrm{Csp}^{3}$ & $\mathrm{C}\left(22^{*}\right)-\mathrm{C}\left(23^{*}\right), \mathrm{C}\left(24^{*}\right)-\mathrm{C}\left(27^{*}\right)$ and $\mathrm{N}\left(3^{*}\right)-\mathrm{C}\left(28^{*}\right)$ & $1.497(5)$ \\
$\mathrm{Csp}^{3}-\mathrm{Csp}^{3}$ & $\mathrm{C}\left(23^{*}\right)-\mathrm{C}\left(24^{*}\right), \mathrm{C}\left(24^{*}\right)-\mathrm{C}\left(25^{*}\right), \mathrm{C}\left(24^{*}\right)-\mathrm{C}\left(26^{*}\right)$ and $\mathrm{C}\left(28^{*}\right)-\mathrm{C}\left(29^{*}\right)$ & $1.538(3)$ \\
$\mathrm{N}=\mathrm{Csp}^{2}$ & $\mathrm{~N}\left(3^{*}\right)-\mathrm{C}\left(27^{*}\right)$ & $1.277(5)$ \\
$\mathrm{S}-\mathrm{Csp} p^{3}$ & $\mathrm{~S}\left(1^{*}\right)-\mathrm{C}\left(29^{*}\right)$ and S$\left(1^{*}\right)-\mathrm{C}\left(30^{*}\right)$ & $1.802(6)$ \\
$\mathrm{Ru}-\mathrm{S}$ & $\mathrm{Ru}^{*}-\mathrm{S}\left(1^{*}\right)$ & $2.561(2)$ \\
$\mathrm{Ru}-\mathrm{Cl}$ & $\mathrm{Ru}^{*}-\mathrm{Cl}\left({ }^{*}\right)$ & $2.409(4)$
\end{tabular}

The anisotropic displacement parameters (ADP's) of the disordered atoms were restrained to simulate isotropic 
behavior. The same restraint was applied to the ADP's of the THF molecules and the occupancy of both were refined together as one value, Occ=0.89 (see Table 1.). Additionally, the ADP's for $C(22 \mathrm{C})$ and $\mathrm{C}(23 \mathrm{C})$ were constrained to be equal. All hydrogen atoms were restrained to ride the corresponding carbon with optimized geometry.

Refinement of $\mathrm{F}^{2}$ against ALL reflections. The weighted R-factor $(w \mathrm{R})$ and goodness of fit (S) are based on $\mathrm{F}^{2}$, conventional R-factors (R) are based on $\mathrm{F}$, with $\mathrm{F}$ set to zero for negative $\mathrm{F}^{2}$. The threshold expression of $\mathrm{F}^{2}>$ $2 \sigma\left(F^{2}\right)$ is used only for calculating R-factors(gt) etc. and is not relevant to the choice of reflections for refinement. $\mathrm{R}$-factors based on $\mathrm{F}^{2}$ are statistically about twice as large as those based on F, and R-factors based on ALL data will be even larger.

All esds (except the esd in the dihedral angle between two l.s. planes) are estimated using the full covariance matrix. The cell esds are taken into account individually in the estimation of esds in distances, angles and torsion angles; correlations between esds in cell parameters are only used when they are defined by crystal symmetry. An approximate (isotropic) treatment of cell esds is used for estimating esds involving l.s. planes. 


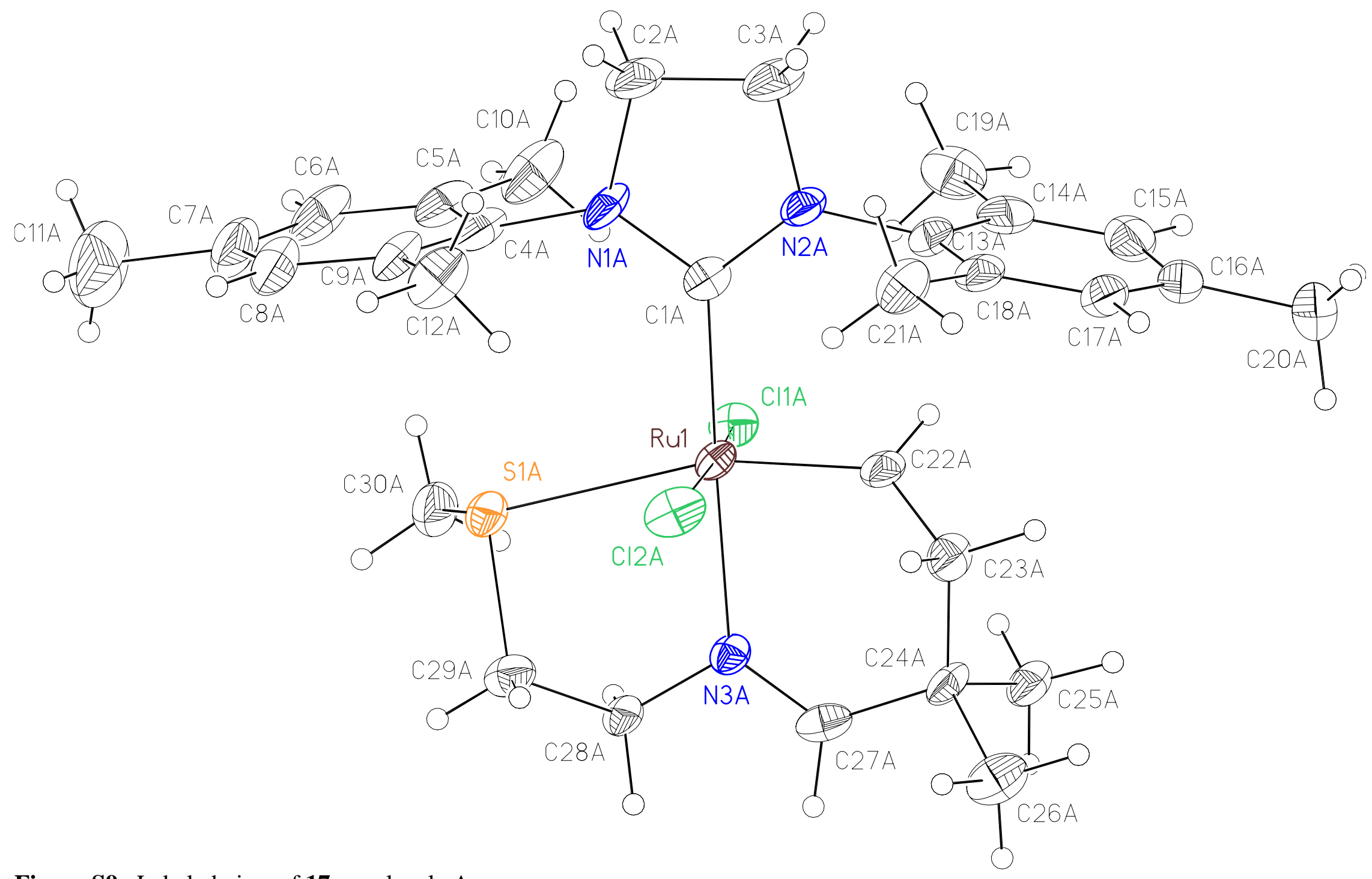

Figure S9. Labeled view of $\mathbf{1 7 c}$, molecule A 


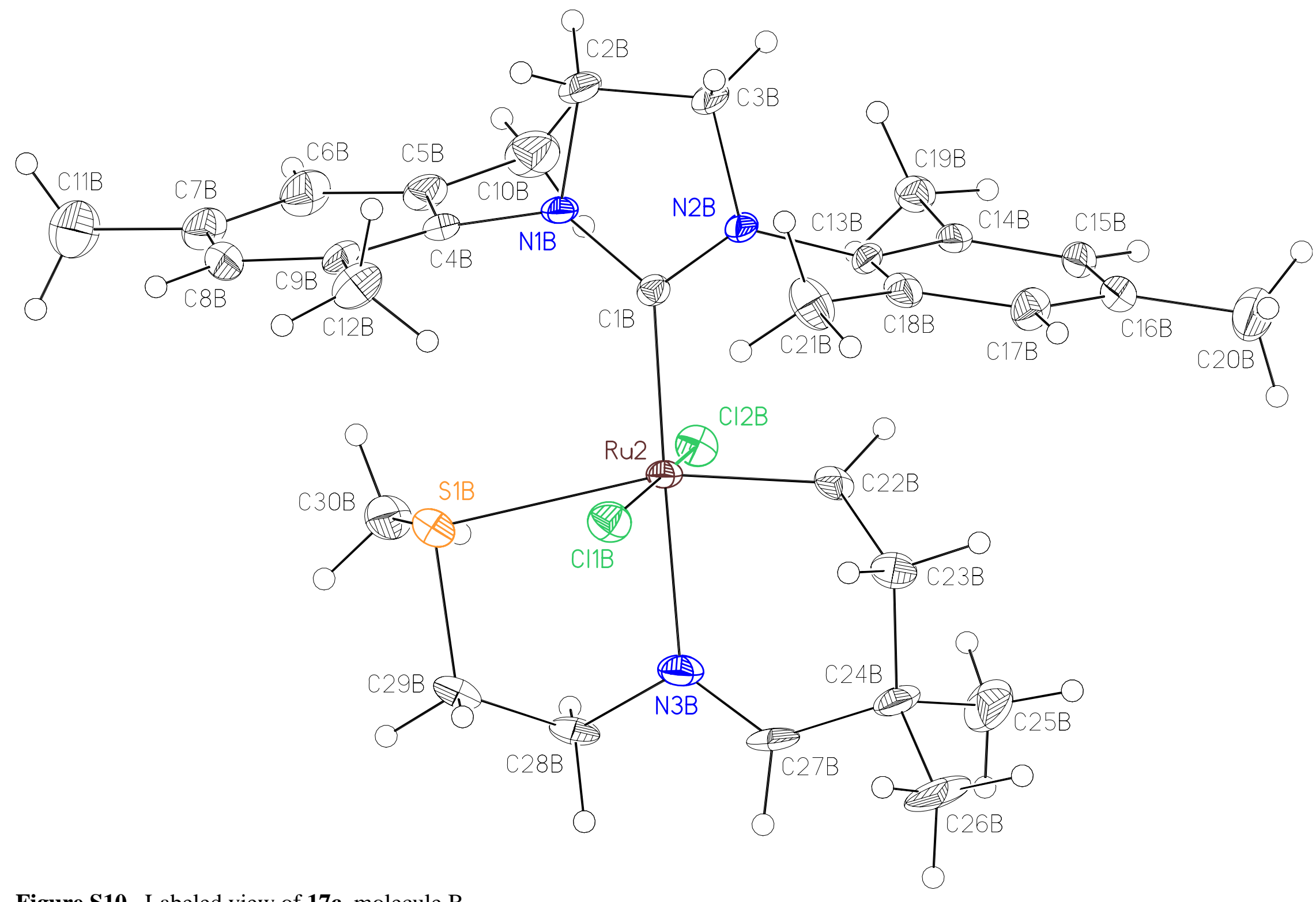

Figure S10. Labeled view of $\mathbf{1 7 c}$, molecule B 


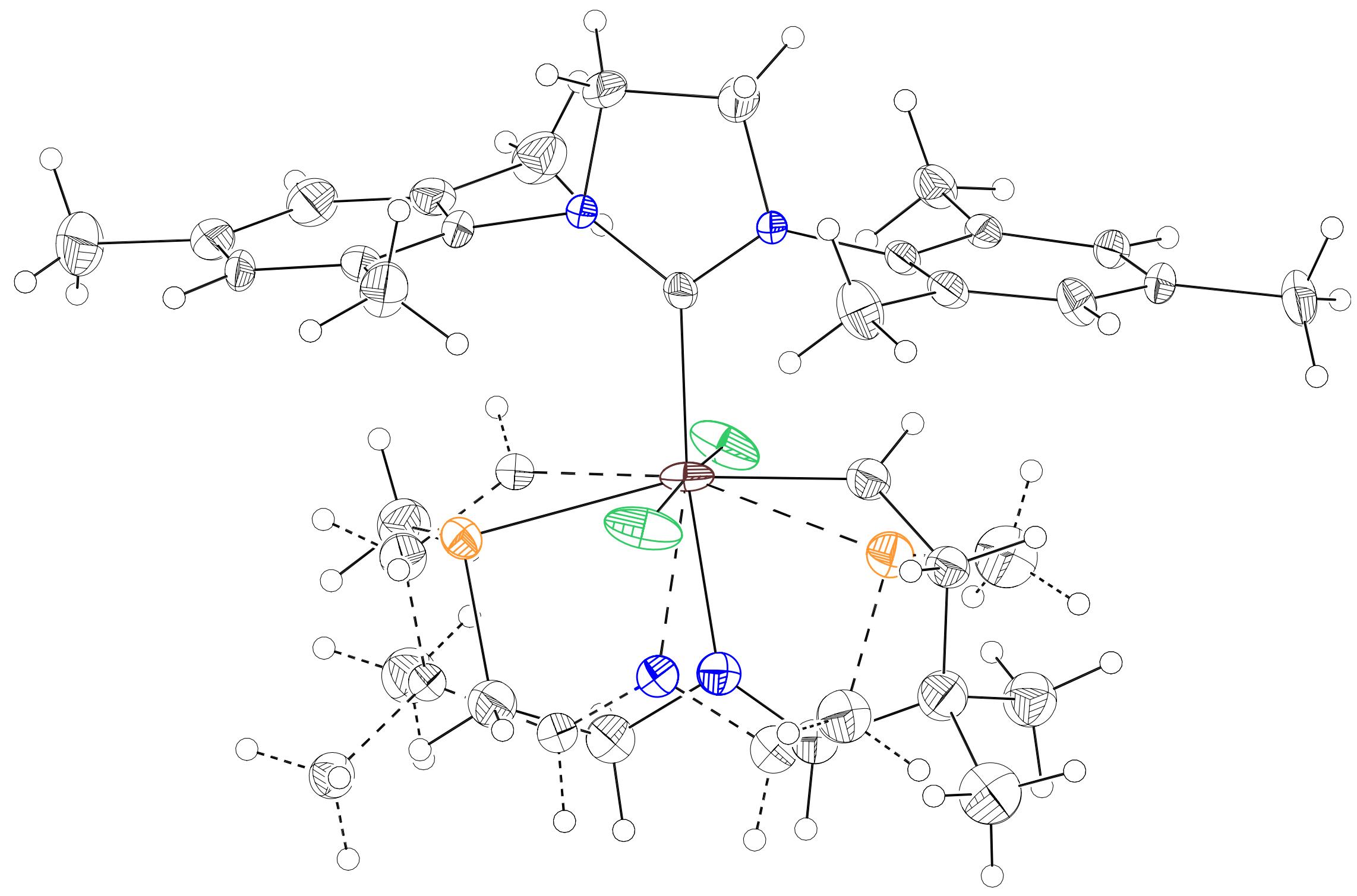

Figure S11. Unlabeled view of 17c, molecule C with minor component (molecule G, dashed lines). 


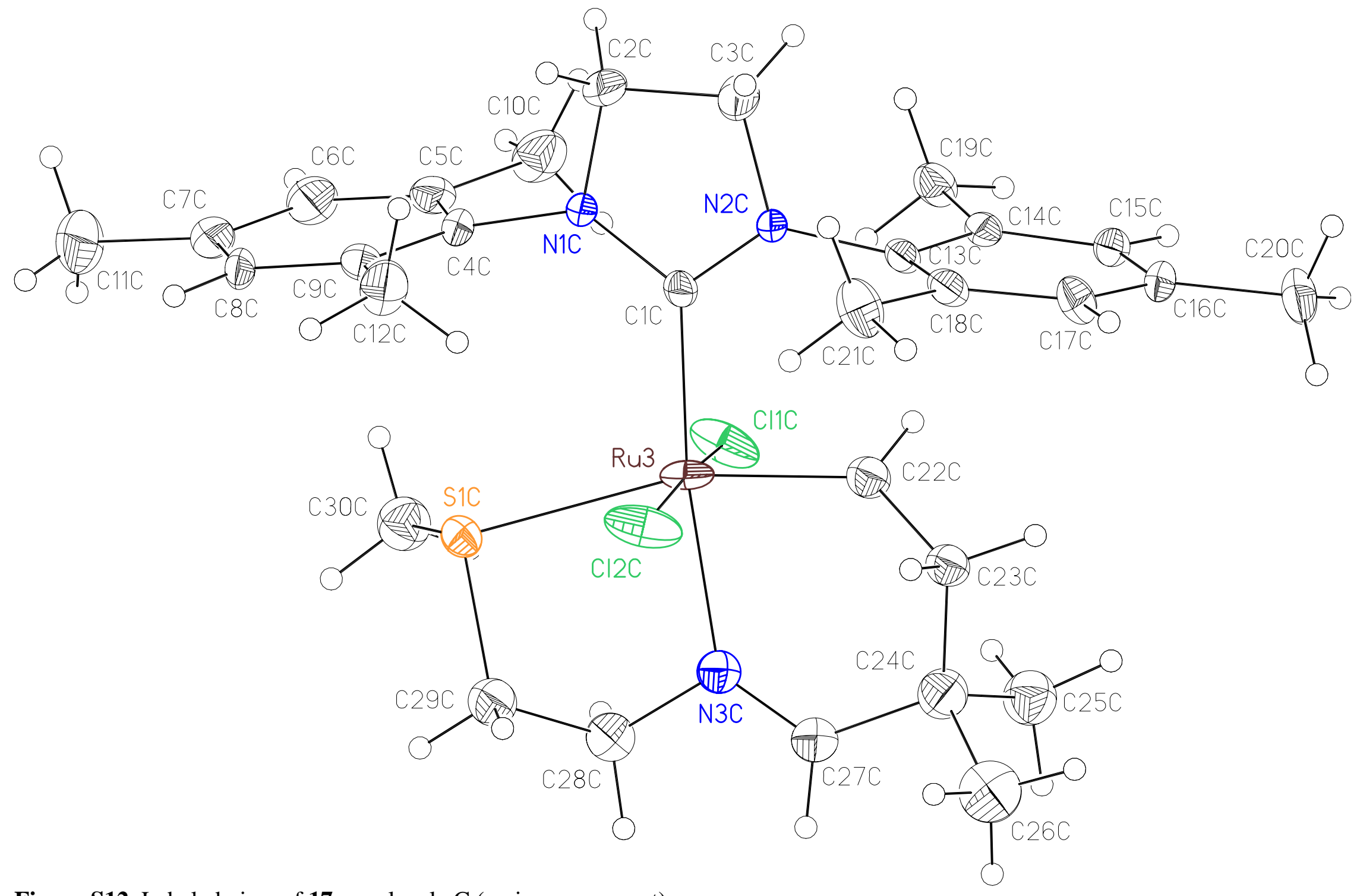

Figure S12. Labeled view of 17c, molecule C (major component). 


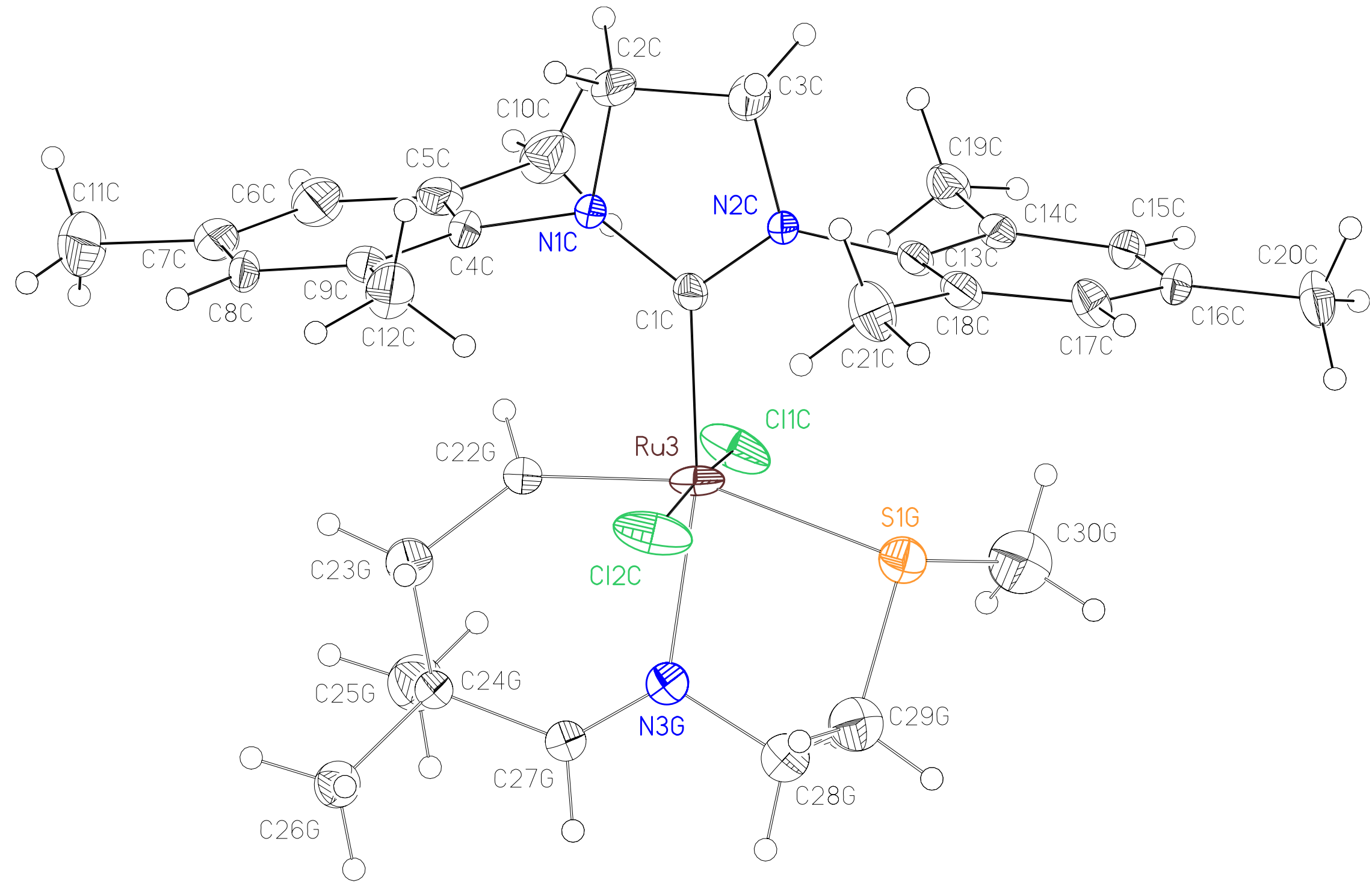

Figure S13. Labeled view of 17c, molecule G (minor component). 


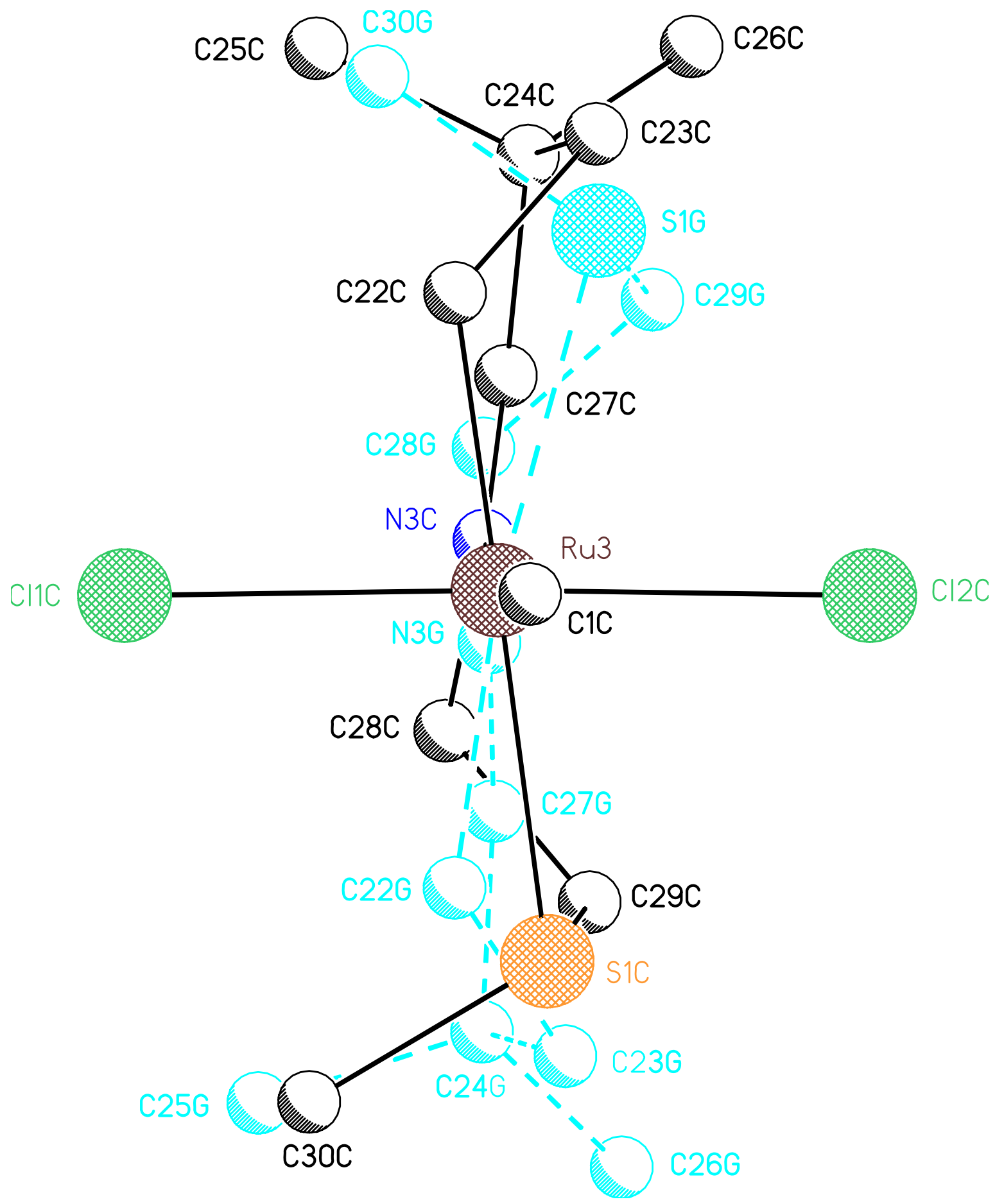

Figure S14. Labeled view of 17c, disordered ligand (hydrogens omitted). The mirror plane is horizontal, normal to the page. The minor component is shown in cyan with dashed bonds. 


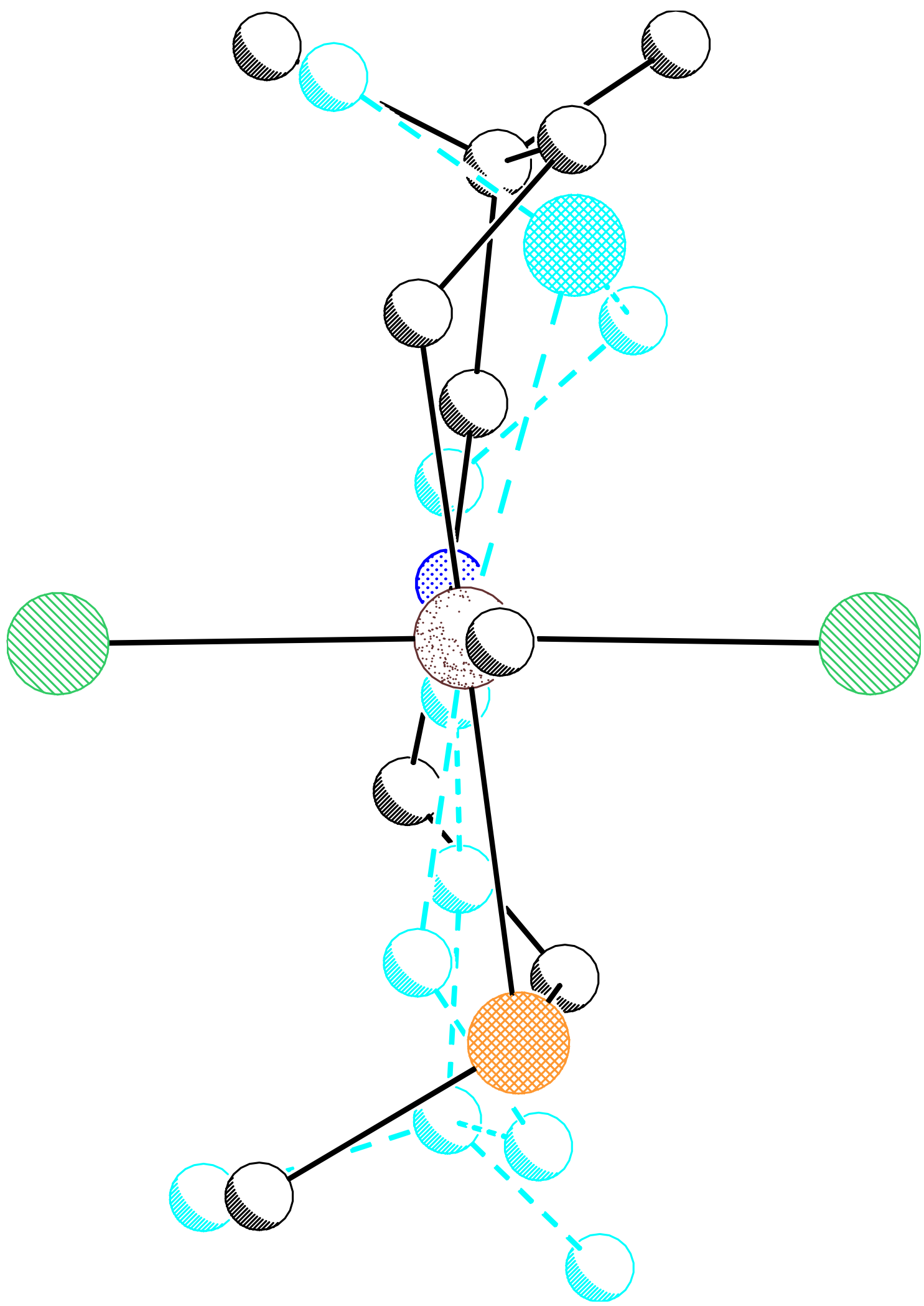

Figure S15. Unlabeled view of 17c disordered ligand (hydrogens omitted). The mirror plane is horizontal, normal to the page. The minor component is shown in cyan with dashed bonds. 


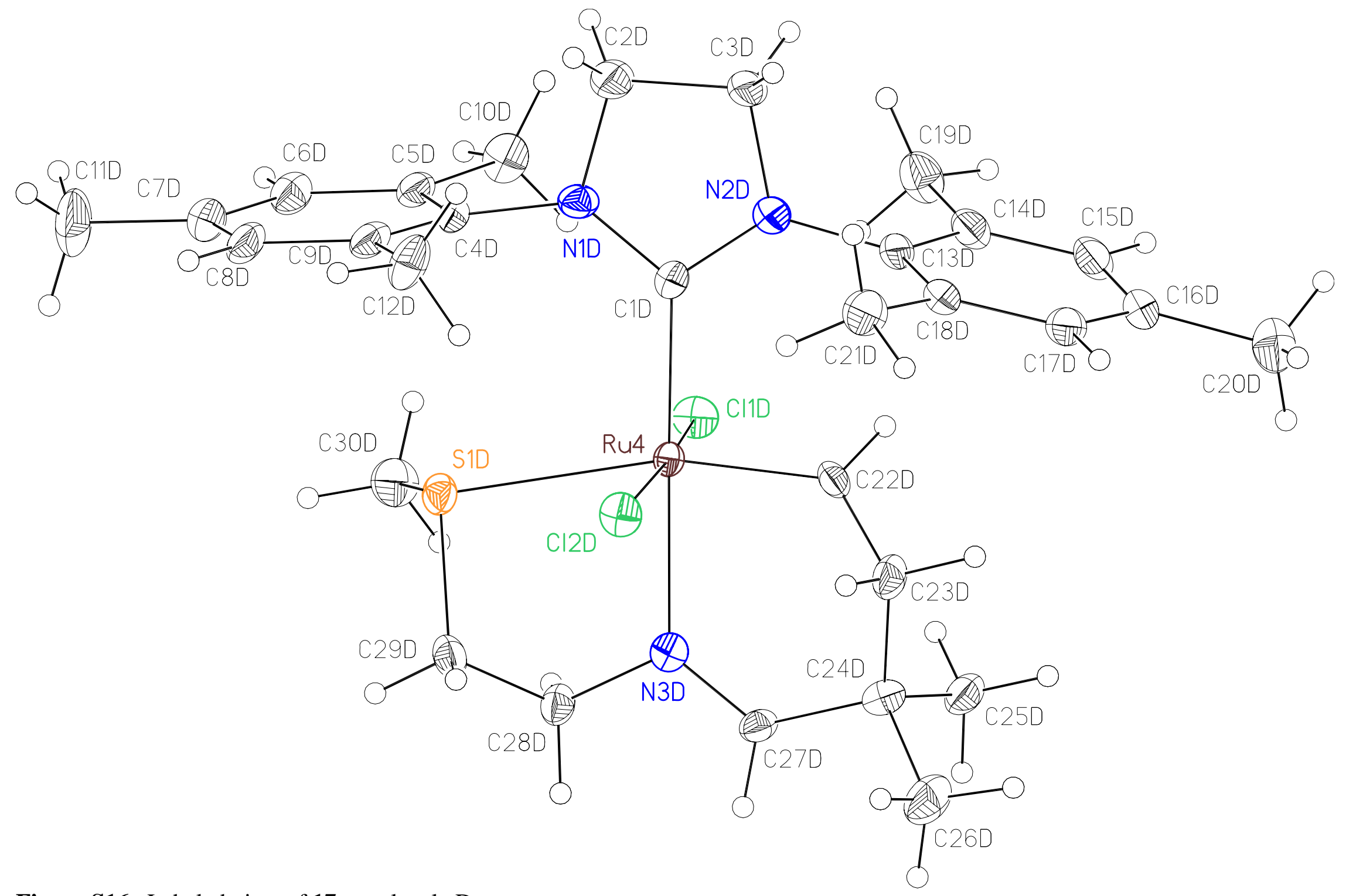

Figure S16. Labeled view of 17c, molecule D 

